\title{
Edge detection with trigonometric polynomial shearlets
}

\section{Kevin Schober ${ }^{1}$ (D) . Jürgen Prestin ${ }^{1}$ - Serhii A. Stasyuk ${ }^{2}$}

Received: 6 February 2020 / Accepted: 17 December 2020 /

Published online: 8 February 2021

(C) The Author(s) 2021

\begin{abstract}
In this paper, we show that certain trigonometric polynomial shearlets which are special cases of directional de la Vallée Poussin-type wavelets are able to detect step discontinuities along boundary curves of periodic characteristic functions. Motivated by recent results for discrete shearlets in two dimensions, we provide lower and upper estimates for the magnitude of the corresponding inner products. In the proof, we use localization properties of trigonometric polynomial shearlets in the time and frequency domain and, among other things, bounds for certain Fresnel integrals. Moreover, we give numerical examples which underline the theoretical results.
\end{abstract}

Keywords Detection of step discontinuities - Trigonometric polynomial shearlets · Directional wavelets · Periodic wavelets

Mathematics Subject Classification (2010) 42C15 · 42C40 · 65T60

\section{Introduction}

In many applications in signal or image processing, great importance is attached to precise information about the location and order of singularities of signals. In

Communicated by: Gitta Kutyniok

Kevin Schober

schober@math.uni-luebeck.de

Jürgen Prestin

prestin@math.uni-luebeck.de

Serhii A. Stasyuk

stasyuk@imath.kiev.ua

1 University of Lübeck, Ratzeburger Allee 160, D-23562, Lübeck, Germany

2 Institute of Mathematics, National Academy of Sciences of Ukraine, 3, Tereschenkivska street, Kyiv, 01024, Ukraine 
one dimension, this corresponds to functions which are smooth apart from pointwise singularities. Many authors discussed this problem when the Fourier coefficients of a periodic function are given; see, e.g., [1, 6, 7, 27, 31, 34].

Because of their localization properties in the time and frequency domain, wavelet expansions provide a powerful tool for detecting and analyzing point discontinuities in one or more dimensions $[18,26]$. The reason is that only very few wavelet coefficients of translates near the location of the singularity are large in magnitude, while all other wavelet coefficients corresponding to translates which are further away from the point discontinuity decay rapidly. A framework for univariate periodic wavelets was investigated by several authors [19, 28-30], and some of these constructions were successfully used for the detection of pointwise singularities of periodic functions [27].

In two dimensions, the situation is more complex since not only point singularities can occur but also discontinuities along curves. To deal with these types of singularities, along with many other constructions, the theory of the continuous shearlet transform was developed $[5,11,22]$ and defined as the mapping:

$$
f \rightarrow \mathcal{S H} \mathcal{H}_{\psi} f(a, s, \mathbf{p})=\left\langle f, \psi_{a, s, \mathbf{p}}\right\rangle
$$

with scale parameter $a>0$, orientation parameter $s \in \mathbb{R}$ and translation parameter $\mathbf{p} \in \mathbb{R}^{2}$. The shearlets $\psi_{a, s, \mathbf{p}}$ are well-localized functions in the time and frequency domain and provide directional sensitivity controlled by the parameter $s$. It turned out that continuous shearlets provide a suitable tool to precisely describe different types of discontinuities along curves with asymptotic estimates. In particular, let $T \subset \mathbb{R}^{2}$ be a set with a smooth boundary $\partial T$. If either $\mathbf{p} \notin \partial T$ or if $s=s_{0}$ does not correspond to the normal direction of $\partial T$ at $\mathbf{p}$, then

$$
\lim _{a \rightarrow 0^{+}} a^{-N} \mathcal{S H}_{\psi} \chi_{T}\left(a, s_{0}, \mathbf{p}\right)=0 \quad \text { for all } N>0 .
$$

Otherwise, if $\mathbf{p} \in \partial T$ and $s=s_{0}$ corresponds to the normal direction of $\partial T$ at $\mathbf{p}$, then

$$
\lim _{a \rightarrow 0^{+}} a^{-3 / 4} \mathcal{S} \mathcal{H}_{\psi} \chi_{T}\left(a, s_{0}, \mathbf{p}\right)=C>0 .
$$

The results were shown for continuous shearlets, which are compactly supported in the time [23] or frequency domain [9, 13, 14, 21].

Based on these theoretical results, practical applications for the detection of edges in images were developed [35]. Therefore, discrete frames of shearlets were constructed by sampling the parameters of the continuous shearlet systems in a suitable way [20]. Based on the result for curvelets [4], it was possible to show that discrete shearlet systems are essentially optimal for the sparse approximation of so-called cartoon-like functions [12]. This result implies the upper estimate:

$$
\left|\left\langle f, \psi_{j, \ell, \mathbf{k}}\right\rangle\right| \leq C 2^{-3 j / 2}
$$

for some constant $C>0$ independent of the scale parameter $j$. In [17], the authors showed the existence of a lower estimate $\left|\left\langle\chi_{T}, \psi_{j, \ell, \mathbf{k}_{\ell}}\right\rangle\right| \geq C 2^{-3 j / 2}$ if the localization and orientation of the discrete shearlet are sufficiently close to the boundary curve and its normal direction. These two estimates are the discrete analogs of Eqs. 1 and 2 
implying that discrete shearlets are able to detect step discontinuities along boundary curves of characteristic functions.

The framework of multivariate periodic wavelets was developed for example in [8, 25]. In [3, 24], the corresponding wavelet functions were trigonometric polynomials of Dirichlet and de la Vallée Poussin-type, which can be well localized in the time and frequency domain. The construction allows for fast decomposition algorithms [2] with many different dilation matrices on each scale, including shearing. This gives rise to directional decompositions of the frequency domain similar to the tilling of the frequency plane in the case of discrete shearlet systems [4, 17].

In this paper, we use the latter construction to prove two main theorems which provide upper and lower bounds similar to [17], but this time for a discrete system of periodic de la Vallée Poussin-type wavelets that are trigonometric polynomials. The upper estimate in Theorem 1 refines the estimate Eq. 3 by including the localization and orientation dependency of the shearlet coefficients in the decay estimate. Theorem 2 is the analog of the main result in [17] and implies that the constructed trigonometric polynomial shearlets in this paper are able to detect step discontinuities along boundary curves of periodic functions.

The paper is organized as follows. We start with the construction of a special case of directional de la Vallée Poussin wavelets in Section 2 which we will call trigonometric polynomial shearlets and state the two main theorems of this paper in Section 3. Section 4 provides a numerical example to illustrate the main results. After some preliminaries, Section 5 is devoted to formulate and to prove all auxiliary lemmata which are needed for the proof of the main results. In Section 6, the proofs for the upper and lower bounds of the corresponding inner products are given. Finally, we consider the extension of the construction to higher dimensions and discuss possible results in the case of corner points and smooth functions.

\section{Trigonometric polynomial shearlets}

If a nonnegative and even function $g: \mathbb{R} \rightarrow \mathbb{R}$ with supp $g=\left(-\frac{2}{3}, \frac{2}{3}\right)$ satisfies the property:

$$
\sum_{z \in \mathbb{Z}} g(x+z)=1 \text { for all } x \in \mathbb{R},
$$

we call it window function and write $g \in \mathcal{W}$. If additionally $g$ is $q$-times continuously differentiable, we use the notation $g \in \mathcal{W}^{q}$. We remark that a consequence of the properties of a window function is $g(x)=1$ for $x \in\left(-\frac{1}{3}, \frac{1}{3}\right)$ and $g$ is monotonically increasing for $x \in\left(-\frac{2}{3},-\frac{1}{3}\right]$ and monotonically decreasing for $x \in\left[\frac{1}{3}, \frac{2}{3}\right)$. Furthermore, we introduce functions $\widetilde{g}: \mathbb{R} \rightarrow \mathbb{R}$ given by $\widetilde{g}(x):=g\left(\frac{x}{2}\right)-g(x)$.

As an example of a window function, we consider:

$$
r(x)= \begin{cases}\mathrm{e}^{-b / x^{2}}, & \text { for } x>0 \\ 0, & \text { for } x \leq 0\end{cases}
$$



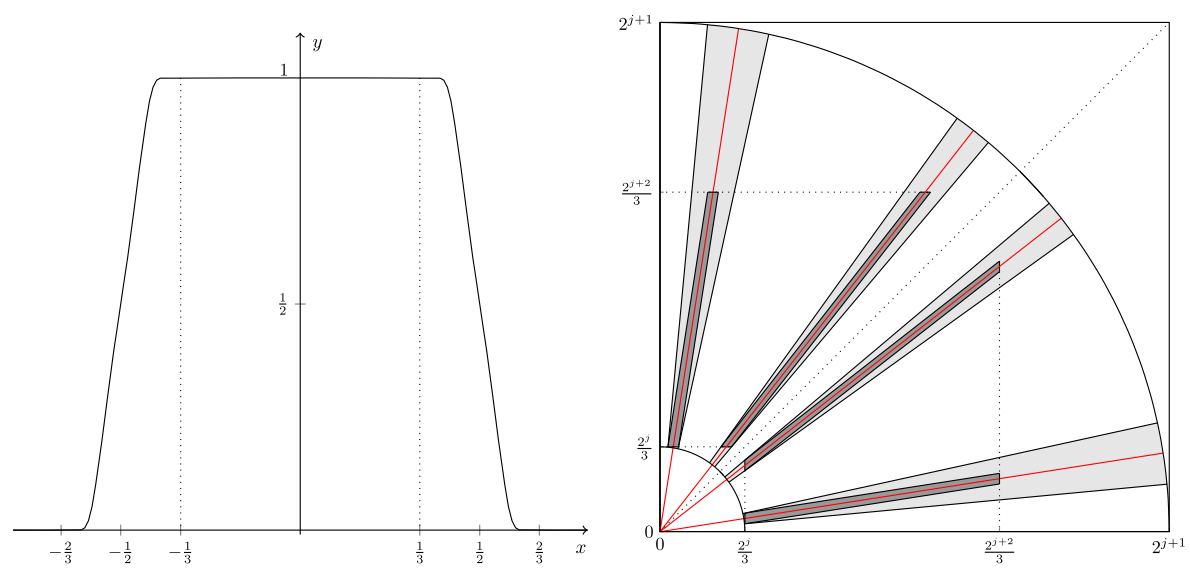

Fig. 1 Left: The window function $g_{0.025} \in \mathcal{W}^{\infty}$ (see Eq. 4). Right: Visualization of supp $\Psi_{10, \ell}^{(i)}$ (dark area) and $W_{10, \ell}^{(i)}$ (light area) for $\ell=5,25$ and $i \in\{\mathrm{h}, \mathrm{v}\}$. The red lines and the horizontal axis form the angles $\theta_{10, \ell}^{(i)}$

where $b>0$ and define $s(x)=r\left(\frac{2}{3}+x\right) r\left(\frac{2}{3}-x\right)$. Then for

$$
g_{b}(x)=\frac{s(x)}{\sum_{k \in \mathbb{Z}} s(x+k)}
$$

we have $g_{b} \in \mathcal{W}^{\infty}$ and this function is visualized in Fig. 1 for $b=0.025$.

We denote two-dimensional vectors by $\mathbf{x}=\left(x_{1}, x_{2}\right)^{\mathrm{T}}$ with the inner product $\mathbf{x}^{\mathrm{T}} \mathbf{y}:=x_{1} y_{1}+x_{2} y_{2}$ and the usual Euclidean norm $|\mathbf{x}|_{2}:=\sqrt{\mathbf{x}^{\mathrm{T}} \mathbf{x}}$. Let $C(A)$ denote the space of all continuous functions on a set $A \subseteq \mathbb{R}^{2}$ equipped with the norm $\|f\|_{A, \infty}:=\|f\|_{C(A)}:=\sup _{\mathbf{x} \in A}|f(\mathbf{x})|$. For $\mathbf{x} \in \mathbb{R}^{2}$ and $\mathbf{r}=\left(r_{1}, r_{2}\right)^{\mathrm{T}} \in \mathbb{N}_{0}^{2}$ and a sufficiently smooth function $f$, we use the notation:

$$
\partial^{\mathbf{r}} f(\mathbf{x}):=\frac{\partial^{r_{1}+r_{2}}}{\partial x_{1}^{r_{1}} \partial x_{2}^{r_{2}}} f(\mathbf{x})
$$

and the space of all $q$-times continuously differentiable compactly supported functions will be denoted by

$C_{0}^{q}(A):=\left\{f: A \rightarrow \mathbb{R}: \partial^{\mathbf{r}} f \in C(A)\right.$ for all $\mathbf{r} \in \mathbb{N}_{0}^{2}$ with $\left.r_{1}+r_{2} \leq q,|\operatorname{supp} f|<\infty\right\}$

with the norm

$$
\|f\|_{C^{q}}:=\|f\|_{C^{q}(A)}:=\sup _{r_{1}+r_{2} \leq q} \sup _{\mathbf{x} \in A}\left|\partial^{\mathbf{r}} f(\mathbf{x})\right| .
$$

For $i \in\{\mathrm{h}, \mathrm{v}\}$, we consider bivariate horizontal (vertical) window functions $\Psi^{(i)}$ : $\mathbb{R}^{2} \rightarrow \mathbb{R}$ given by

$$
\Psi^{(\mathrm{h})}(\mathbf{x}):=\widetilde{g}\left(x_{1}\right) g\left(x_{2}\right), \quad \Psi^{(\mathrm{v})}(\mathbf{x}):=g\left(x_{1}\right) \widetilde{g}\left(x_{2}\right) .
$$


We remark that for $g \in \mathcal{W}^{q}$ we have $\Psi^{(i)} \in C_{0}^{q}\left(\mathbb{R}^{2}\right)$ and in this case use the notation $\Psi^{(i)} \in \mathcal{W}_{2}^{q}$. From the support properties of the function $g \in \mathcal{W}$, it follows that:

$$
\begin{aligned}
& \operatorname{supp} \Psi^{(\mathrm{h})}=\left(\left(-\frac{4}{3},-\frac{1}{3}\right) \cup\left(\frac{1}{3}, \frac{4}{3}\right)\right) \times\left(-\frac{2}{3}, \frac{2}{3}\right), \\
& \operatorname{supp} \Psi^{(\mathrm{v})}=\left(-\frac{2}{3}, \frac{2}{3}\right) \times\left(\left(-\frac{4}{3},-\frac{1}{3}\right) \cup\left(\frac{1}{3}, \frac{4}{3}\right)\right) .
\end{aligned}
$$

For even $j \in \mathbb{N}_{0}$ and $\ell \in \mathbb{Z}$ with $|\ell| \leq 2^{j / 2}$, we define the matrices:

$$
\mathbf{N}_{j, \ell}^{(\mathrm{h})}:=\left(\begin{array}{cc}
2^{j} & \ell 2^{j / 2} \\
0 & 2^{j / 2}
\end{array}\right), \quad \quad \mathbf{N}_{j, \ell}^{(\mathrm{v})}:=\left(\begin{array}{cc}
2^{j / 2} & 0 \\
\ell 2^{j / 2} & 2^{j}
\end{array}\right)
$$

and the corresponding discrete angles

$$
\theta_{j, \ell}^{(\mathrm{h})}:=\arctan \left(\ell 2^{-j / 2}\right), \quad \theta_{j, \ell}^{(\mathrm{v})}:=\operatorname{arccot}\left(\ell 2^{-j / 2}\right) .
$$

Note that these matrices occur in the construction of discrete shearlet systems, for example in $[12,17]$. Based on this, we introduce the notation:

$$
\Psi_{j, \ell}^{(i)}(\cdot):=\Psi^{(i)}\left(\left(\mathbf{N}_{j, \ell}^{(i)}\right)^{-\mathrm{T}} \cdot\right)
$$

and, since $\operatorname{det} \mathbf{N}_{j, \ell}^{(i)}=2^{3 j / 2}$, it follows that

$$
\left|\operatorname{supp} \Psi_{j, \ell}^{(i)}\right|=\left|\operatorname{supp} \Psi^{(i)}\right| \operatorname{det} \mathbf{N}_{j, \ell}^{(i)}=\frac{8}{3} 2^{3 j / 2} .
$$

In polar coordinates, we define the sets

$$
\begin{aligned}
& W_{j, \ell}^{(\mathrm{h})}:=\left\{(\rho, \theta) \in \mathbb{R} \times\left[-\frac{\pi}{2}, \frac{\pi}{2}\right]: \frac{2^{j}}{3}<|\rho|<2^{j+1}, \theta_{j, \ell-2}^{(\mathrm{h})}<\theta<\theta_{j, \ell+2}^{(\mathrm{h})}\right\}, \\
& W_{j, \ell}^{(\mathrm{v})}:=\left\{(\rho, \theta) \in \mathbb{R} \times[0, \pi]: \frac{2^{j}}{3}<|\rho|<2^{j+1}, \theta_{j, \ell+2}^{(\mathrm{v})}<\theta<\theta_{j, \ell-2}^{(\mathrm{v})}\right\}
\end{aligned}
$$

and based on ideas from [12, Proposition 2.1] we show the following lemma, which is visualized on the right side of Fig. 1.

Lemma 1 For even $j \geq 10, \ell \in \mathbb{Z}$ with $|\ell| \leq 2^{j / 2}$ and $i \in\{\mathrm{h}, \mathrm{v}\}$, we have

$$
\operatorname{supp} \Psi_{j, \ell}^{(i)}(\rho, \theta) \subset W_{j, \ell}^{(i)}
$$

Proof We show only the case $i=\mathrm{h}$ since the other one is similar. In Eq. 6, we defined

$$
\Psi_{j, \ell}^{(\mathrm{h})}(\boldsymbol{\xi})=\Psi^{(\mathrm{h})}\left(\left(\mathbf{N}_{j, \ell}^{(\mathrm{h})}\right)^{-\mathrm{T}} \boldsymbol{\xi}\right)=\tilde{g}\left(2^{-j} \xi_{1}\right) g\left(2^{-j} \xi_{1}\left(2^{j / 2} \frac{\xi_{2}}{\xi_{1}}-\ell\right)\right)
$$

with the support property

$$
\operatorname{supp} \widetilde{g}\left(2^{-j} \xi_{1}\right)=\left\{\xi_{1} \in \mathbb{R}: \frac{2^{j}}{3}<\left|\xi_{1}\right|<\frac{2^{j+2}}{3}\right\}
$$


and, assuming that $\xi_{1} \in \operatorname{supp} \widetilde{g}\left(2^{-j} \cdot\right)$, we have

$$
\begin{aligned}
\operatorname{supp} g\left(2^{-j} \xi_{1}\left(2^{j / 2} \frac{\xi_{2}}{\xi_{1}}-\ell\right)\right) & =\left\{\xi_{2} \in \mathbb{R}:\left|2^{-j} \xi_{1}\left(2^{j / 2} \frac{\xi_{2}}{\xi_{1}}-\ell\right)\right|<\frac{2}{3}\right\} \\
& =\left\{\xi_{2} \in \mathbb{R}:\left|\ell-2^{j / 2} \frac{\xi_{2}}{\xi_{1}}\right|<\frac{2^{j+1}}{3\left|\xi_{1}\right|}\right\} \\
& \subset\left\{\xi_{2} \in \mathbb{R}:\left|\ell-2^{j / 2} \frac{\xi_{2}}{\xi_{1}}\right|<2\right\} .
\end{aligned}
$$

In the following, we introduce polar coordinates with the notation $\boldsymbol{\xi}:=\rho \boldsymbol{\Theta}(\theta)$, where $\boldsymbol{\Theta}(\theta):=(\cos \theta, \sin \theta)^{\mathrm{T}}$. Recalling the discrete angles $\theta_{j, \ell}^{(\mathrm{h})}=\arctan \left(\ell 2^{-j / 2}\right)$ we have

$$
\begin{aligned}
\operatorname{supp} g\left(2^{-j} \xi_{1}\left(2^{j / 2} \frac{\xi_{2}}{\xi_{1}}-\ell\right)\right) & \subset\left\{\theta \in\left[-\frac{\pi}{2}, \frac{\pi}{2}\right]:\left|\ell-2^{j / 2} \tan \theta\right|<2\right\} \\
& =\left\{\theta \in\left[-\frac{\pi}{2}, \frac{\pi}{2}\right]: \theta_{j, \ell-2}^{(\mathrm{h})}<\theta<\theta_{j, \ell+2}^{(\mathrm{h})}\right\} .
\end{aligned}
$$

Since $\rho^{2}=\xi_{1}^{2}\left(1+\tan ^{2} \theta\right)$ and $|\ell| \leq 2^{j / 2}$ we can show

$$
|\rho| \leq \frac{2^{j+2}}{3}\left(1+2^{-j}(|\ell|+2)^{2}\right)^{1 / 2} \leq \frac{2^{j+2}}{3}\left(2+2^{2-j / 2}+2^{2-j}\right)^{1 / 2}<2^{j+1},
$$

where the last inequality holds for $j \geq 10$. As a lower bound for the radius $\rho$, we obtain:

$$
|\rho| \geq \frac{2^{j}}{3}\left(1+2^{-j}(|\ell|+2)^{2}\right)^{1 / 2}>\frac{2^{j}}{3} .
$$

The pattern of a regular matrix $\mathbf{M} \in \mathbb{Z}^{2 \times 2}$ is defined by $\mathcal{P}(\mathbf{M}):=\mathbf{M}^{-1} \mathbb{Z}^{2} \cap$ $\left[-\frac{1}{2}, \frac{1}{2}\right)^{2}$. As a consequence of [24, Lemma 2.4], the patterns of the matrices in Eq. 5 are independent of the parameter $\ell$ and have the tensor product structure:

$$
\begin{aligned}
\mathcal{P}\left(\mathbf{N}_{j, \ell}^{(\mathrm{h})}\right)= & \left\{2^{-j} z_{1}: z_{1}=-2^{j-1}, \ldots, 2^{j-1}-1\right\} \\
& \times\left\{2^{-j / 2} z_{2}: z_{2}=-2^{j / 2-1}, \ldots, 2^{j / 2-1}-1\right\}, \\
\mathcal{P}\left(\mathbf{N}_{j, \ell}^{(\mathrm{v})}\right)= & \left\{2^{-j / 2} z_{1}: z_{1}=-2^{j / 2-1}, \ldots, 2^{j / 2-1}-1\right\} \\
& \times\left\{2^{-j} z_{2}: z_{2}=-2^{j-1}, \ldots, 2^{j-1}-1\right\} .
\end{aligned}
$$

For $i \in\{\mathrm{h}, \mathrm{v}\}$ and $\Psi^{(i)} \in \mathcal{W}_{2}^{q}$ the translates of the de la Vallee Poussin wavelet functions (see [3]) on the pattern points $\mathbf{y} \in \mathcal{P}\left(\mathbf{N}_{j, \ell}^{(i)}\right)$ are trigonometric polynomials given by

$$
\psi_{j, \ell, \mathbf{y}}^{(i)}(\mathbf{x}):=\sum_{\mathbf{k} \in \mathbb{Z}^{2}} \Psi_{j, \ell}^{(i)}(\mathbf{k}) \mathrm{e}^{\mathrm{i} \mathbf{k}^{\mathrm{T}}(\mathbf{x}-2 \pi \widetilde{\mathbf{y}})}
$$

where

$$
\tilde{\mathbf{y}}:= \begin{cases}\mathbf{y}-\left(2^{-j-1}, 0\right)^{\mathrm{T}}, & \text { for } \mathbf{y} \in \mathcal{P}\left(\mathbf{N}_{j, \ell}^{(\mathrm{h})}\right) \\ \mathbf{y}-\left(0,2^{-j-1}\right)^{\mathrm{T}}, & \text { for } \mathbf{y} \in \mathcal{P}\left(\mathbf{N}_{j, \ell}^{(\mathrm{v})}\right)\end{cases}
$$


In the following, we call the functions $\psi_{j, \ell, \mathbf{y}}^{(i)}$ trigonometric polynomial shearlets.

\section{Main results}

Let $\rho(t):[0,2 \pi) \rightarrow[0, \pi)$ fulfilling

$$
\sup _{0 \leq t<2 \pi}|\rho \prime \prime(t)| \leq \kappa<\infty
$$

and let $\gamma:[0,2 \pi) \rightarrow(-\pi, \pi)^{2}$ be a closed curve with

$$
\boldsymbol{\gamma}(t):=\rho(t)\left(\begin{array}{c}
\cos t \\
\sin t
\end{array}\right), \quad t \in[0,2 \pi)
$$

which is a parametrization of the boundary of a set $T \subset(-\pi, \pi)^{2}$. The space $\mathcal{C}^{u}(\kappa)$ is defined as the collection of all functions of the form

$$
f=f_{0}+f_{1} \chi_{T}
$$

where $f_{0}, f_{1} \in C^{u}\left([-\pi, \pi]^{2}\right), u \geq 2$.

Following the ideas from $[4,12]$, let $\mathcal{Q}_{j}, j \in \mathbb{N}_{0}$, be the set of dyadic squares $Q \subseteq[-\pi, \pi)^{2}$ of the form (Fig. 2)

$$
\begin{aligned}
Q= & {\left[2 \pi n_{1} 2^{-j / 2}-\pi, 2 \pi\left(n_{1}+1\right) 2^{-j / 2}-\pi\right) } \\
& \times\left[2 \pi n_{2} 2^{-j / 2}-\pi, 2 \pi\left(n_{2}+1\right) 2^{-j / 2}-\pi\right)
\end{aligned}
$$

with $n_{1}, n_{2}=0, \ldots, 2^{j / 2}-1$. Let $Q \in \mathcal{Q}_{j}^{1} \subseteq \mathcal{Q}_{j}$ if $\partial T \cap Q \neq \emptyset$ and for the nonintersecting squares we define $\mathcal{Q}_{j}^{0}:=\mathcal{Q}_{j} \backslash \mathcal{Q}_{j}^{1}$. We remark that $\left|\mathcal{Q}_{j}^{0}\right| \leq C 2^{j}$ and $\left|\mathcal{Q}_{j}^{1}\right| \leq C_{2} 2^{j / 2}$ (see $\left.[4,12]\right)$.

For Lebesgue measurable sets $A \subseteq \mathbb{R}^{2}$ and functions $f: A \rightarrow \mathbb{R}$ define

$$
\|f\|_{A, p}:=\left(\int_{A}|f(\mathbf{x})|^{p} \mathrm{~d} \mathbf{x}\right)^{1 / p}, \quad 1 \leq p<\infty,
$$

and let $L_{p}(A)$ denote the collection of functions satisfying $\|f\|_{A, p}<\infty$. In particular, two-dimensional $2 \pi$-periodic functions $f: \mathbb{T}^{2} \rightarrow \mathbb{R}$ are defined on the torus $\mathbb{T}^{2}:=\mathbb{R}^{2} \backslash 2 \pi \mathbb{Z}^{2}$. Recall that the usual inner product of the Hilbert space $L_{2}\left(\mathbb{T}^{2}\right)$ is given by:

$$
\langle f, g\rangle_{2}:=(2 \pi)^{-2} \int_{\mathbb{T}^{2}} f(\mathbf{x}) \overline{g(\mathbf{x})} \mathrm{d} \mathbf{x}, \quad f, g \in L_{2}\left(\mathbb{T}^{2}\right),
$$

and for $f \in L_{1}\left(\mathbb{R}^{2}\right)$ we call

$$
f^{2 \pi}:=\sum_{\mathbf{n} \in \mathbb{Z}^{2}} f(\cdot+2 \pi \mathbf{n})
$$

the $2 \pi$-periodization of $f$.

The main results of this paper are stated in the following two theorems. 

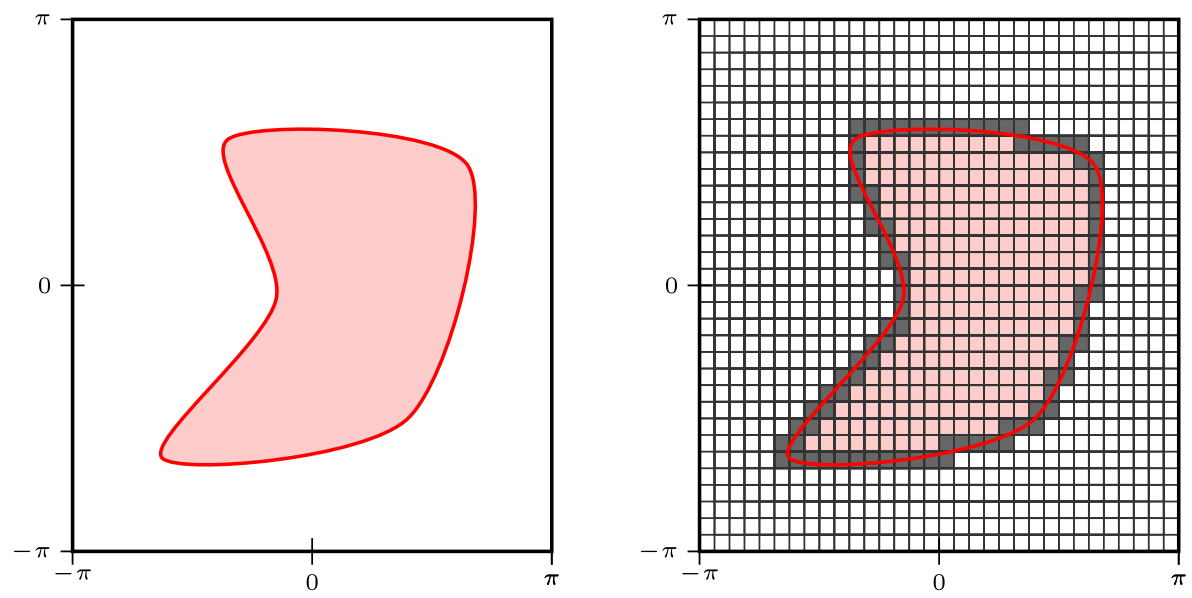

Fig. 2 Left: Characteristic function of a set $T \subset(-\pi, \pi)^{2}$ with boundary $\partial T$. Right: Decomposition into dyadic squares for $j=10$, where $Q \in \mathcal{Q}_{j}^{0}$ are colored white and $Q \in \mathcal{Q}_{j}^{1}$ along $\partial T$ are colored dark

Theorem 1 Let $f \in \mathcal{C}^{2}(\kappa)$ and $\Psi^{(i)} \in \mathcal{W}_{2}^{2 q}, i \in\{\mathrm{h}, \mathrm{v}\}$ for $q \geq 2$. Moreover for $Q \in \mathcal{Q}_{j}^{1}$ let $\mathbf{x}_{0}:=\mathbf{x}_{0}(Q) \in \partial T \cap Q$ and $\gamma:=\gamma\left(\mathbf{x}_{0}\right)$ such that $(\cos \gamma, \sin \gamma)^{\mathrm{T}}$ is the normal direction of the boundary curve $\partial T$ in $\mathbf{x}_{0}$. Then, we have:

$$
\left|\left\langle f^{2 \pi}, \psi_{j, \ell, \mathbf{y}}^{(i)}\right\rangle_{2}\right| \leq C(q) \sum_{Q \in \mathcal{Q}_{j}^{1}}\left(1+2^{j}\left|\mathbf{x}_{0}-2 \pi \widetilde{\mathbf{y}}\right|_{2}^{2}\right)^{-q}\left(1+2^{j / 2}\left|\sin \left(\theta_{j, \ell}^{(i)}-\gamma\right)\right|\right)^{-5 / 2} .
$$

If $\mathbf{y} \in \mathcal{P}\left(\mathbf{N}_{j, \ell}^{(i)}\right)$ is sufficiently far away from the boundary curve, Theorem 1 implies

$$
\left|\left\langle f^{2 \pi}, \psi_{j, \ell, \mathbf{y}}^{(i)}\right\rangle_{2}\right| \leq C(q) 2^{-j(q-1 / 2)} .
$$

For the special case $f_{0}=0$ and $f_{1}=1$ in Eq. 8 we define $\mathcal{T}=\chi_{T}$ and denote by $\mathcal{T}^{2 \pi}$ the $2 \pi$-periodization of $\mathcal{T}$.

Theorem 2 Let $\Psi^{(i)} \in \mathcal{W}_{2}^{2 q}$ for sufficiently large $q \in \mathbb{N}$ and $\mathbf{y} \in \mathcal{P}\left(\mathbf{N}_{j, \ell}^{(i)}\right)$ for large $j$. If there exists $\mathbf{x}_{0} \in \partial T$ with the normal direction $(\cos \gamma, \sin \gamma)^{\mathrm{T}}$ and curvature $A_{0}$ in that point, fulfilling $\left|\mathbf{x}_{0}-2 \pi \widetilde{\mathbf{y}}\right|_{2} \leq C 2^{-j / 2}$ and $\theta_{j, \ell}^{(i)} \leq \gamma \leq \theta_{j, \ell+1}^{(i)}$ for $i \in\{\mathrm{h}, \mathrm{v}\}$, then there is a constant $C\left(q, A_{0}\right)>0$ such that

$$
\left|\left\langle\mathcal{T}^{2 \pi}, \psi_{j, \ell, \mathbf{y}}^{(i)}\right\rangle_{2}\right| \geq C\left(q, A_{0}\right) .
$$

\section{Numerical examples}

In this section, we give numerical examples to underline the main results of this paper by computing the shearlet coefficients of a characteristic function of a rotated ellipse. In order to do that, we need to compute the Fourier transform of the characteristic 
function of a disc, given by

$$
D(\mathbf{x}):= \begin{cases}1 & \text { for }|\mathbf{x}|_{2} \leq 1 \\ 0 & \text { else }\end{cases}
$$

We transform $\boldsymbol{\xi}=\rho \boldsymbol{\Theta}(\theta)$ and $\mathbf{x}=r \boldsymbol{\Theta}(\phi)$ into polar coordinates and use $\boldsymbol{\xi}^{\mathrm{T}} \mathbf{x}=$ $r \rho \cos (\theta-\phi)$ to obtain

$$
\begin{aligned}
\mathcal{F}[D](\xi) & =\frac{1}{(2 \pi)^{2}} \int_{\mathbb{R}^{2}} D(\mathbf{x}) \mathrm{e}^{-\mathrm{i} \xi^{\mathrm{T}} \mathbf{x}} \mathrm{d} \mathbf{x} \\
& =\frac{1}{(2 \pi)^{2}} \int_{0}^{1} \int_{0}^{2 \pi} \mathrm{e}^{-\mathrm{i} r \rho \cos (\theta-\phi)} r \mathrm{~d} \phi \mathrm{d} r=\frac{1}{2 \pi} \int_{0}^{1} r J_{0}(r \rho) \mathrm{d} r,
\end{aligned}
$$

where $J_{0}$ is the Bessel function of the first kind and zero order. The integral identity

$$
\int_{0}^{u} t J_{0}(t) \mathrm{d} t=u J_{1}(u)
$$

together with the change of variable $\lambda=r \rho$ leads to

$$
\mathcal{F}[D](\xi)=\frac{1}{2 \pi} \int_{0}^{1} r J_{0}(r \rho) \mathrm{d} r=\frac{1}{2 \pi \rho^{2}} \int_{0}^{\rho} \lambda J_{0}(\lambda) \mathrm{d} \lambda=\frac{J_{1}(\rho)}{2 \pi \rho}=\frac{J_{1}\left(|\boldsymbol{\xi}|_{2}\right)}{2 \pi|\boldsymbol{\xi}|_{2}} .
$$

For $a, b>0$, we define $D_{a, b}(\mathbf{x}):=D\left(a^{-1} x_{1}, b^{-1} x_{2}\right)$ and convert the circle into a characteristic function of an ellipse with major semi-axis of length $a$ and minor semi-axis of length $b$. By the scaling property of the Fourier transform, we have:

$$
\mathcal{F}\left[D_{a, b}\right](\xi)=\frac{a b J_{1}\left(\left|\left(a \xi_{1}, b \xi_{2}\right)\right|_{2}\right)}{2 \pi\left|\left(a \xi_{1}, b \xi_{2}\right)\right|_{2}} .
$$

If we further rotate the function $D_{a, b}$ by an angle $\gamma \in[0,2 \pi)$ we obtain a rotated ellipse $D_{a, b, \gamma}(\mathbf{x}):=D_{a, b}\left(\mathbf{R}_{\gamma} \mathbf{x}\right)$ and its Fourier transform is given by $\mathcal{F}\left[D_{a, b, \gamma}\right](\xi)=\mathcal{F}\left[D_{a, b}\right]\left(\mathbf{R}^{\mathrm{T}} \boldsymbol{\xi}\right)$

In order to calculate the shearlet coefficients of a rotated ellipse, we consider the $2 \pi$-periodized function $D_{a, b, \gamma}^{2 \pi}(\mathbf{x})$. We use Eq. 15 to see that the Fourier coefficients of this function are given by

$$
c_{\mathbf{k}}\left(D_{a, b, \gamma}^{2 \pi}\right)=\mathcal{F}\left[D_{a, b, \gamma}\right](\mathbf{k}), \mathbf{k} \in \mathbb{Z}^{2},
$$

and Parseval's identity finally gives

$$
\left\langle D_{a, b, \gamma}^{2 \pi}, \psi_{j, \ell, \mathbf{y}}^{(i)}\right\rangle_{2}=\sum_{\mathbf{k} \in \mathbb{Z}^{2}} \mathcal{F}\left[D_{a, b, \gamma}\right](\mathbf{k}) \Psi_{j, \ell}^{(i)}(\mathbf{k}) \mathrm{e}^{2 \pi \mathrm{i} \mathbf{k}^{\mathrm{T}} \widetilde{\mathbf{y}}} .
$$

In our numerical example, we calculate the inner product Eq. 11 with Mathematica 12. We fix the characteristic function of the rotated ellipse $D_{1,3, \frac{\pi}{6}}^{2 \pi}$, which is depicted on the left side in Fig. 3 (gray area). For the one-dimensional window function, we 

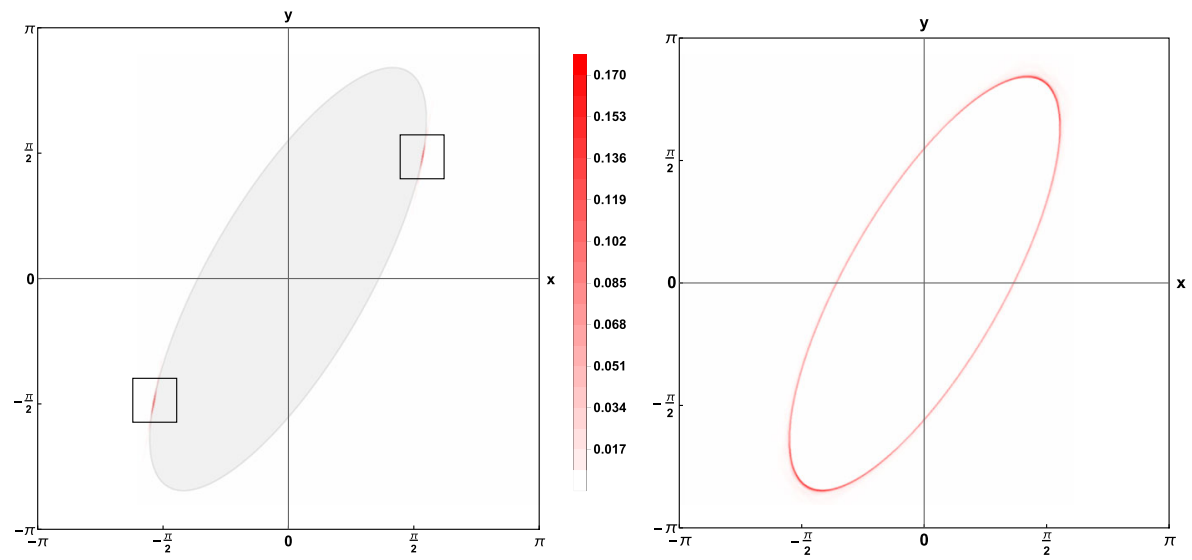

Fig. 3 Left: Characteristic function $D_{1,3, \frac{\pi}{6}}^{2 \pi}$ (gray) and magnitude of the inner product $\left|\left\langle D_{1,3, \frac{\pi}{6}}^{2 \pi}, \psi_{10,-3, \mathbf{y}}^{(\mathrm{h})}\right\rangle_{2}\right|$ (red) for every $\mathbf{y} \in \mathcal{P}\left(\mathbf{M}_{10}\right)$. Right: Magnitude of $\sum_{\ell=-2^{j / 2}+1}^{2^{j / 2}-1}\left|\left\langle D_{1,3, \frac{\pi}{6}}^{2 \pi}, \psi_{10, \ell, \mathbf{y}}^{(i)}\right\rangle_{2}\right|$ for every $\mathbf{y} \in \mathcal{P}\left(\mathbf{M}_{10}\right)$ and $i \in\{\mathrm{h}, \mathrm{v}\}$.

use the smooth function $g_{0.025} \in \mathcal{W}^{\infty}$ constructed in Eq. 4 . We fix the scale $j=10$ and for a better visualization we consider the matrix $\mathbf{M}_{10}=2^{10} \mathbf{I}_{2}$, where $\mathbf{I}_{2}$ is the two-dimensional identity matrix and compute the shearlet coefficients in Eq. 11 on the pattern $\mathcal{P}\left(\mathbf{M}_{10}\right)$, which corresponds to a two-dimensional equidistant grid of $1024 \times 1024$ points. Thus, the images in this example are of size $1024 \times 1024$, where every pixel corresponds to the magnitude of the inner product with a translate of the trigonometric polynomial shearlet $\psi_{j, \ell, \mathbf{y}}^{(i)}, \mathbf{y} \in \mathcal{P}\left(\mathbf{M}_{10}\right)$.

On the left side of Fig. 3, we set the directional parameter to $\ell=-3$ and observe that the magnitude of the coefficients

$$
\left|\left\langle D_{1,3, \frac{\pi}{6}}^{2 \pi}, \psi_{10,-3, \mathbf{y}}^{(\mathrm{h})}\right\rangle_{2}\right|
$$

is very close to zero except for the pattern points $\mathbf{y} \in \mathcal{P}\left(\mathbf{M}_{10}\right)$, for which the function $\psi_{10,-3, \mathbf{y}}^{(\mathrm{h})}$ is close to points $\mathbf{x} \in \partial D_{1,3, \frac{\pi}{6}}^{2 \pi}$ on the boundary with normal direction almost parallel to the direction induced by the angle $\theta_{10,-3}^{(\mathrm{h})}$.

To make this more clear, the left image of Fig. 4 zooms into the upper right black square. The dotted line is parallel to the line, which forms the angle $\theta_{10,-3}^{(\mathrm{h})}$ with the horizontal axis. One can observe that the only significant shearlet coefficients are close to the boundary and nearly orthogonal to this line. In other words, only if the trigonometric shearlet $\psi_{10,-3, \mathbf{y}}^{(\mathrm{h})}$ is almost aligned with the boundary $\partial D_{1,3, \frac{\pi}{6}}^{2 \pi}$, the inner product Eq. 11 yields large values. The right graph in Fig. 4 is a logarithmic plot of the shearlet coefficients $\left|\left\langle D_{1,3, \frac{\pi}{6}}^{2 \pi}, \psi_{10,-3, \mathbf{y}}^{(\mathrm{h})}\right\rangle_{2}\right|$ for every $\mathbf{y} \in \mathcal{P}\left(\mathbf{M}_{10}\right)$ in descending order. The red part of the line corresponds to the coefficients visible in the left picture of Fig. 3. 

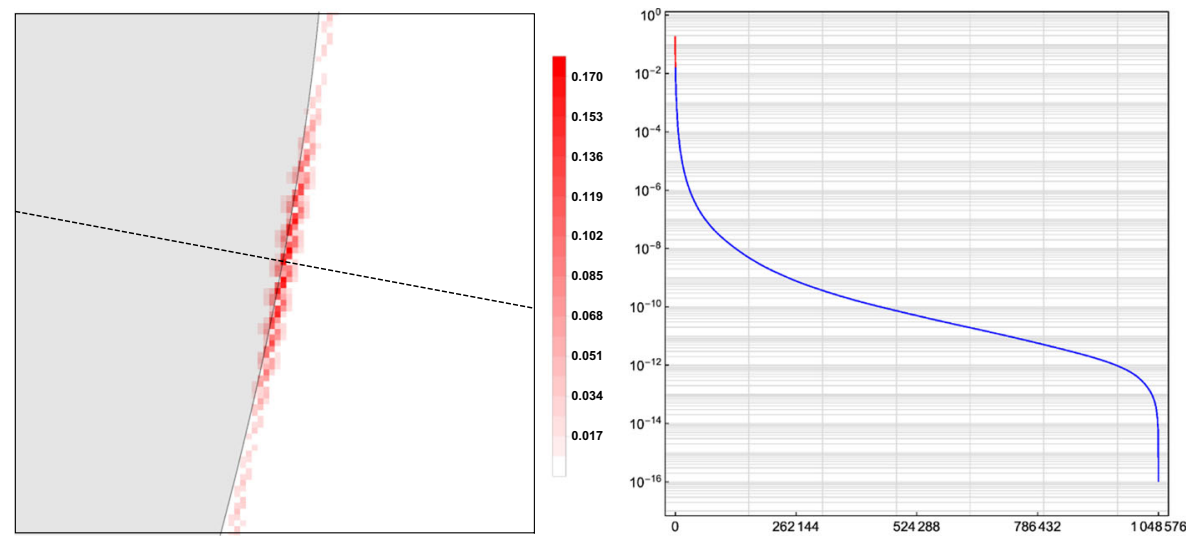

Fig. 4 Left: Zoom into the upper right window from the left image of Fig. 3. Left: Logarithmic plot of the shearlet coefficients $\left|\left\langle D_{1,3, \frac{\pi}{6}}^{2 \pi}, \psi_{10,-3, \mathbf{y}}^{(\mathrm{h})}\right\rangle_{2}\right|$ for every $\mathbf{y} \in \mathcal{P}\left(\mathbf{M}_{10}\right)$ in descending order

The right image illustrates the capability of the trigonometric polynomial shearlets to detect step discontinuities along the boundary of characteristic functions. For $i \in$ $\{\mathrm{h}, \mathrm{v}\}$ and $\ell=-2^{j / 2}+1, \ldots, 2^{j / 2}-1$, we compute all the pictures of the shearlet coefficients similar to the left image in Fig. 3 and add them component-wise to get the final result. Thus, every pixel of the image is given by the sum:

$$
\sum_{\ell=-2^{j / 2}+1}^{2^{j / 2}-1}\left|\left\langle D_{1,3, \frac{\pi}{6}}^{2 \pi}, \psi_{10, \ell, \mathbf{y}}^{(i)}\right\rangle_{2}\right|, \quad i \in\{\mathrm{h}, \mathrm{v}\}, \mathbf{y} \in \mathcal{P}\left(\mathbf{M}_{10}\right),
$$

and one can clearly see the only significant coefficients for all the directions are exact on the boundary of $D_{1,3, \frac{\pi}{6}}^{2 \pi}$.

Besides the visual representations of the detection of step discontinuities with trigonometric polynomial shearlets, we want to illustrate the upper and lower estimates given in the two main theorems. In order to do so for the upper bound, we compute the quantity:

$$
U_{j, \ell}^{(i)}:=\max _{\mathbf{y} \in \mathcal{P}\left(\mathbf{M}_{j}\right)} \frac{\left|\left\langle D_{1,3, \frac{\pi}{6}}^{2 \pi}, \psi_{j, \ell, \mathbf{y}}^{(i)}\right\rangle_{2}\right|}{\sum_{Q \in \mathcal{Q}_{j}^{1}}\left(1+2^{j}\left|\mathbf{x}_{0}-2 \pi \tilde{\mathbf{y}}\right|_{2}^{2}\right)^{-q}\left(1+2^{j / 2}\left|\sin \left(\theta_{j, \ell}^{(i)}-\gamma\right)\right|\right)^{-5 / 2}}
$$

In the left graph of Fig. 5, the values $U_{j, \ell}^{(i)}$ are plotted for different orientation angles $\theta_{j, \ell}^{(i)}$. One can see that the quotient $U_{j, \ell}^{(i)}$ is bounded from above by a moderate constant for every $j$ and $\ell$ which confirms that the estimate in Theorem 1 provides a valid upper bound.

For the lower bound, we collect all pattern points $\mathbf{y} \in \mathcal{P}\left(\mathbf{M}_{j}\right)$ for which there exists $\mathbf{x}_{0} \in \partial T$ with the normal direction $(\cos \gamma, \sin \gamma)^{\mathrm{T}}$ fulfilling $\left|\mathbf{x}_{0}-2 \pi \widetilde{\mathbf{y}}\right|_{2} \leq$ 

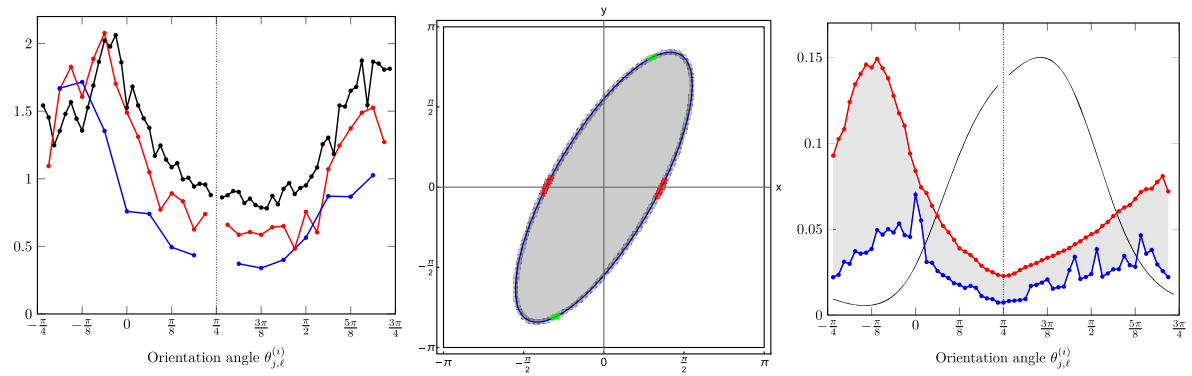

Fig. 5 Left: $U_{j, \ell}^{(i)}$ for $j=4$ (blue line), $j=6$ (red line) and $j=8$ (black line). Middle: $L_{j, \ell}^{(i)}$ for $j=8$ and $\ell \in \mathbb{Z}$ with $|\ell|<2^{j / 2}$ (dark points), $L_{8,-8}^{(\mathrm{h})}$ (red points) and $L_{8,-8}^{(\mathrm{v})}$ (green points). Right: $L_{j, \ell}^{(i), \max }$ (red line), $L_{j, \ell}^{(i), \min }$ (blue line) and the curvature values $\frac{1}{20} \kappa(x)$ (black line)

$C 2^{-j / 2}$ and $\theta_{j, \ell}^{(i)} \leq \gamma \leq \theta_{j, \ell+1}^{(i)}$ and call this set $L_{j, \ell}^{(i)}$. As an example, the set $L_{j, \ell}^{(i)}$ is shown in the middle of Fig. 5 for $j=8$ and all $\ell \in \mathbb{Z}$ with $|\ell|<2^{j / 2}$ together with $L_{8,-8}^{(\mathrm{h})}$ and $L_{8,-8}^{(\mathrm{v})}$. The latter two sets include pattern points which are close to boundary points $\mathbf{x}_{0} \in \partial T$ with normal direction $(\cos \gamma, \sin \gamma)^{\mathrm{T}}$ fulfilling $\theta_{8,-8}^{(i)} \leq \gamma \leq \theta_{8,-7}^{(i)}$ for $i \in\{\mathrm{h}, \mathrm{v}\}$. By Theorem 2, we expect the values of the shearlet coefficients corresponding to the shearlets $\psi_{8,-8, \mathbf{y}}^{(i)}$ for $\mathbf{y} \in L_{8,-8}^{(i)}$ to be bounded from below by a constant. Therefore, we compute the values

$$
L_{j, \ell}^{(i), \max }:=\max _{\mathbf{y} \in L_{j, \ell}^{(i)}}\left|\left\langle D_{1,3, \frac{\pi}{6}}^{2 \pi}, \psi_{j, \ell, \mathbf{y}}^{(i)}\right\rangle_{2}\right|, \quad L_{j, \ell}^{(i), \min }:=\min _{\mathbf{y} \in L_{j, \ell}^{(i)}}\left|\left\langle D_{1,3, \frac{\pi}{6}}^{2 \pi}, \psi_{j, \ell, \mathbf{y}}^{(i)}\right\rangle_{2}\right|
$$

and show them in the right graph of Fig. 5 as functions of the orientation angles $\theta_{j, \ell}^{(i)}$. One can clearly see that the minimal values $L_{j, \ell}^{(i), \text { min }}$ are bounded from below, which confirms the result of Theorem 2. In our numerical example, a parametrization of the boundary $\partial D_{1,3, \frac{\pi}{6}}$ is given by $\gamma(x)=\frac{1}{2}(\sqrt{3} \cos x+3 \sin x, 3 \sqrt{3} \sin x-\cos x)^{\mathrm{T}}$ and the curvature in each point is given by $\kappa(x)=3(5+4 \cos (2 x))^{-3 / 2}$. On the right side of Fig. 5, the values of the curvature $\kappa(x)$ are shown in the points $\mathbf{x}_{0} \in \partial T$, where the normal direction of $\mathbf{x}_{0}$ is orthogonal to $\theta_{j, \ell}^{(i)}$ for $|\ell|<2^{j / 2}$. As anticipated in Theorem 2, one can see that the magnitude of the coefficients $L_{j, \ell}^{(i), \text { max }}, L_{j, \ell}^{(i) \text {,min }}$ varies as the curvature of the ellipse changes. If the curvature is small, which corresponds to the "stretched" part of the boundary, the values become larger. Intuitively, this makes sense since in that case a large part of the boundary is aligned with the corresponding shearlet. 


\section{Auxiliary results}

For two-dimensional vector norms, we use the notation:

$$
|\mathbf{x}|_{p}:= \begin{cases}\left(\left|x_{1}\right|^{p}+\left|x_{2}\right|^{p}\right)^{1 / p}, & \text { if } 1 \leq p<\infty, \\ \max \left\{\left|x_{1}\right|,\left|x_{2}\right|\right\}, & \text { if } p=\infty\end{cases}
$$

and for binary relations and exponentials of vectors we write $\mathbf{x} \leq \mathbf{y}$ if $x_{1} \leq y_{1}$ and $x_{2} \leq y_{2}, \mathbf{x}^{\mathbf{y}}:=x_{1}^{y_{1}} x_{2}^{y_{2}}$ and $\mathbf{x}^{\beta}:=\mathbf{x}^{\beta \mathbf{1}}=x_{1}^{\beta} x_{2}^{\beta}$ for $\beta \in \mathbb{R}$. Moreover for $\mathbf{k}, \mathbf{n} \in \mathbb{N}_{0}^{2}$ with $\mathbf{k} \leq \mathbf{n}$ and $n \in \mathbb{N}_{0}$ with $\mathbf{k} \leq n \mathbf{1}$ we define $\mathbf{k} !:=k_{1} ! k_{2}$ ! and

$$
\left(\begin{array}{l}
\mathbf{n} \\
\mathbf{k}
\end{array}\right):=\frac{\mathbf{n} !}{\mathbf{k} !(\mathbf{n}-\mathbf{k}) !}=\left(\begin{array}{l}
n_{1} \\
k_{1}
\end{array}\right)\left(\begin{array}{l}
n_{2} \\
k_{2}
\end{array}\right), \quad\left(\begin{array}{l}
n \\
\mathbf{k}
\end{array}\right):=\frac{n !}{\mathbf{k} !\left(n-|\mathbf{k}|_{1}\right) !} .
$$

The Fourier coefficients of a function $f \in L_{1}\left(\mathbb{T}^{2}\right)$ are given by

$$
c_{\mathbf{k}}(f):=(2 \pi)^{-2} \int_{\mathbb{T}^{2}} f(\mathbf{x}) \mathrm{e}^{-\mathrm{i} \mathbf{k}^{\mathrm{T}} \mathbf{x}} \mathrm{d} \mathbf{x}, \quad \mathbf{k} \in \mathbb{Z}^{2} .
$$

The Fourier transform of $f \in L_{1}\left(\mathbb{R}^{2}\right)$ is defined as

$$
\mathcal{F}[f](\mathbf{x}):=\mathcal{F} f(\mathbf{x}):=(2 \pi)^{-2} \int_{\mathbb{R}^{2}} f(\boldsymbol{\xi}) \mathrm{e}^{-\mathrm{i} \xi^{\mathrm{T}} \mathbf{x}} \mathrm{d} \boldsymbol{\xi}, \quad \mathbf{x} \in \mathbb{R}^{2},
$$

and we have the operator

$$
\mathcal{F}^{-1}[f](\mathbf{x}):=\mathcal{F}^{-1} f(\mathbf{x}):=\int_{\mathbb{R}^{2}} f(\boldsymbol{\xi}) \mathrm{e}^{\mathrm{i} \xi^{\mathrm{T}} \mathbf{x}} \mathrm{d} \boldsymbol{\xi}, \quad \mathbf{x} \in \mathbb{R}^{2} .
$$

For $f \in L_{1}\left(\mathbb{R}^{2}\right)$ and $\mathcal{F} f \in L_{1}\left(\mathbb{R}^{2}\right)$, the inversion formula $f(\mathbf{x})=\mathcal{F F}^{-1} f(\mathbf{x})=$ $\mathcal{F}^{-1} \mathcal{F} f(\mathbf{x})$ holds for all $\mathbf{x} \in \mathbb{R}^{2}$. We recall some basic results about the Fourier transform and its connection to Fourier series via the Poisson summation formula. Let $q \in \mathbb{N}_{0}$ and $\mathbf{r} \in \mathbb{N}_{0}^{2}$ with $|\mathbf{r}|_{1} \leq q$. If $f \in L_{1}\left(\mathbb{R}^{2}\right)$ and (i $\left.\mathbf{x}\right)^{q} f \in L_{1}\left(\mathbb{R}^{2}\right)$, then $\mathcal{F} f \in C^{q}\left(\mathbb{R}^{2}\right)$ and

$$
\partial^{\mathbf{r}} \mathcal{F} f(\boldsymbol{\xi})=\mathcal{F}\left[(\mathrm{i} \mathbf{x})^{\mathbf{r}} f(\mathbf{x})\right](\boldsymbol{\xi}) .
$$

Moreover for $f \in C^{q}\left(\mathbb{R}^{2}\right)$ and $\partial^{\mathbf{r}} f \in L_{1}\left(\mathbb{R}^{2}\right)$, we have

$$
\mathcal{F}\left[\partial^{\mathbf{r}} f\right](\xi)=(\mathrm{i} \xi)^{\mathbf{r}} \mathcal{F} f(\xi) .
$$

It is well known that there are constants $C_{1}(q, f), C_{2}(q, f)>0$ such that for $f \in$ $C_{0}^{q}\left(\mathbb{R}^{2}\right)$ with $q \in \mathbb{N}_{0}$ and all $\mathbf{x} \in \mathbb{R}^{2}$, we have:

$$
|\mathcal{F} f(\mathbf{x})| \leq \frac{C_{1}(q, f)}{\left(1+|\mathbf{x}|_{2}\right)^{q}}, \quad\left|\mathcal{F}^{-1} f(\mathbf{x})\right| \leq \frac{C_{2}(q, f)}{\left(1+|\mathbf{x}|_{2}\right)^{q}} .
$$

The sum in Eq. 10 converges for almost every $\mathbf{x} \in \mathbb{T}^{2}$ and $f^{2 \pi} \in L_{1}\left(\mathbb{T}^{2}\right)$. For the Fourier coefficients, we have:

$$
c_{\mathbf{k}}\left(f^{2 \pi}\right)=\mathcal{F} f(\mathbf{k}), \quad \mathbf{k} \in \mathbb{Z}^{2} .
$$

It is a consequence of Eq. 14 and [33, Corollary VII.2.6] that for a function $f \in$ $C_{0}^{q}\left(\mathbb{R}^{2}\right)$ with $q>2$, the Poisson summation formula

$$
\sum_{\mathbf{k} \in \mathbb{Z}^{2}} \mathcal{F} f(\mathbf{k}) \mathrm{e}^{\mathrm{i} \mathbf{k}^{\mathrm{T}} \mathbf{x}}=\sum_{\mathbf{n} \in \mathbb{Z}^{2}} f(\mathbf{x}+2 \pi \mathbf{n})=f^{2 \pi}(\mathbf{x})
$$


holds true for all $\mathbf{x} \in \mathbb{R}^{2}$.

In the following, we prepare the proof of Theorem 1 with several auxiliary lemmata. Note that in the proofs we only show the case $i=\mathrm{h}$ since the other case can be handled similarly.

Lemma 2 For $i \in\{\mathrm{h}, \mathrm{v}\}$ and $q \in \mathbb{N}_{0}$ let $\Psi^{(i)} \in \mathcal{W}_{2}^{q}$ be given. Then for $\mathbf{r} \in \mathbb{N}_{0}^{2}$ with $|\mathbf{r}|_{1} \leq q$ and a rotation matrix $\mathbf{R}_{\gamma}$ with $\gamma \in[0,2 \pi)$ we have

$$
\begin{aligned}
\left|\partial^{\mathbf{r}} \Psi_{j, \ell}^{(i)}\left(\mathbf{R}_{\gamma} \boldsymbol{\xi}\right)\right| \leq & C(q) 2^{-j|\mathbf{r}|_{1}}\left(1+2^{(j+1) / 2}\left|\sin \left(\theta_{j, \ell}^{(i)}-\gamma\right)\right|\right)^{r_{1}} \\
& \times\left(1+2^{(j+1) / 2}\left|\cos \left(\theta_{j, \ell}^{(i)}-\gamma\right)\right|\right)^{r_{2}} .
\end{aligned}
$$

Proof We have $\mathbf{R}_{\gamma}=\left(\begin{array}{cc}\cos \gamma & -\sin \gamma \\ \sin \gamma & \cos \gamma\end{array}\right)$ and use Eq. 6 to see

$$
\begin{aligned}
\Psi_{j, \ell}^{(\mathrm{h})}\left(\mathbf{R}_{\gamma} \boldsymbol{\xi}\right)=g & \left(2^{-j / 2}\left(\xi_{1} \sin \gamma+\xi_{2} \cos \gamma\right)-\ell 2^{-j}\left(\xi_{1} \cos \gamma-\xi_{2} \sin \gamma\right)\right) \\
& \times \widetilde{g}\left(2^{-j}\left(\xi_{1} \cos \gamma-\xi_{2} \sin \gamma\right)\right) .
\end{aligned}
$$

In this proof, we will omit the long arguments of the function of the last line and simply write $g$ and $\widetilde{g}$. For $\mathbf{m}=\left(m_{1}, m_{2}\right)^{\mathrm{T}}$ with $|\mathbf{m}|_{1} \leq q$, we use the chain rule to get

$$
\left|\partial^{\mathbf{m}} \widetilde{g}\right|=\|\widetilde{g}\|_{C^{q}} 2^{-j|\mathbf{m}|_{1}}|\cos \gamma|^{m_{1}}|\sin \gamma|^{m_{2}} \leq C(q) 2^{-j|\mathbf{m}|_{1}}
$$

and, since $\ell=2^{j / 2} \tan \left(\theta_{j, \ell}^{(\mathrm{h})}\right)$, we have

$$
\begin{aligned}
\mid \partial^{\mathbf{m}_{g} \mid} & =\|g\|_{C^{q}}\left|2^{-j / 2} \sin \gamma-\ell 2^{-j} \cos \gamma\right|^{m_{1}}\left|2^{-j / 2} \cos \gamma+\ell 2^{-j} \sin \gamma\right|^{m_{2}} \\
& =C_{2}(q) 2^{-j|\mathbf{m}|_{1}}\left(2^{j / 2} \frac{\left|\sin \left(\theta_{j, \ell}^{(\mathrm{h})}-\gamma\right)\right|}{\left|\cos \left(\theta_{j, \ell}^{(\mathrm{h})}\right)\right|}\right)^{m_{1}}\left(2^{j / 2} \frac{\left|\cos \left(\theta_{j, \ell}^{(\mathrm{h})}-\gamma\right)\right|}{\left|\cos \left(\theta_{j, \ell}^{(\mathrm{h})}\right)\right|}\right)^{m_{2}} .
\end{aligned}
$$

For sufficiently smooth functions $f, g: \mathbb{R}^{2} \rightarrow \mathbb{R}$, we employ the multivariate Leibniz rule

$$
\partial^{\mathbf{r}}(f g)=\sum_{\mathbf{0} \leq \mathbf{s} \leq \mathbf{r}}\left(\begin{array}{l}
\mathbf{r} \\
\mathbf{s}
\end{array}\right) \partial^{\mathbf{s}} f \partial^{\mathbf{r}-\mathbf{s}} g,
$$

which together with the triangle inequality and the binomial theorem implies

$$
\begin{aligned}
\left|\partial^{\mathbf{r}} \Psi_{j, \ell}^{(\mathrm{h})}\left(\mathbf{R}_{\gamma} \boldsymbol{\xi}\right)\right| \leq & C_{3}(q) 2^{-j|\mathbf{r}|_{1}} \sum_{\mathbf{0} \leq \mathbf{s} \leq \mathbf{r}}\left(\begin{array}{l}
\mathbf{r} \\
\mathbf{s}
\end{array}\right)\left(2^{j / 2} \frac{\left|\sin \left(\theta_{j, \ell}^{(\mathrm{h})}-\gamma\right)\right|}{\left|\cos \left(\theta_{j, \ell}^{(\mathrm{h})}\right)\right|}\right)^{s_{1}} \\
& \times\left(2^{j / 2} \frac{\left|\cos \left(\theta_{j, \ell}^{(\mathrm{h})}-\gamma\right)\right|}{\left|\cos \left(\theta_{j, \ell}^{(\mathrm{h})}\right)\right|}\right) \\
\leq & C_{4}(q) 2^{-j|\mathbf{r}|_{1}}\left(1+2^{(j+1) / 2}\left|\sin \left(\theta_{j, \ell}^{(i)}-\gamma\right)\right|\right)^{r_{1}} \\
& \times\left(1+2^{(j+1) / 2}\left|\cos \left(\theta_{j, \ell}^{(i)}-\gamma\right)\right|\right)^{r_{2}}
\end{aligned}
$$


since $2^{-1 / 2} \leq\left|\cos \left(\theta_{j, \ell}^{(\mathrm{h})}\right)\right| \leq 1$.

In the following, we use notations and ideas from [4, 12] and fix a function $\phi \in$ $C_{0}^{\infty}\left([-\pi, \pi]^{2}\right)$. Denote $\phi_{j}(\mathbf{x}):=\phi\left(2^{j / 2} \mathbf{x}\right)$ and for $Q \in \mathcal{Q}_{j}$ given by Eq. 9 we define

$$
\phi_{Q}(\mathbf{x}):=\phi\left(2^{j / 2}\left(x_{1}+\pi\right)-\pi\left(2 k_{1}-1\right), 2^{j / 2}\left(x_{2}+\pi\right)-\pi\left(2 k_{2}-1\right)\right)
$$

for $k_{1}, k_{2}=1, \ldots, 2^{j / 2}$ and assume that $\phi$ defines a smooth partition of unity

$$
\sum_{Q \in \mathcal{Q}_{j}} \phi_{Q}(\mathbf{x})=1, \quad \mathbf{x} \in[-\pi, \pi)^{2} .
$$

The ideas of the proof of the next lemma can be found in $[4,12]$.

Lemma 3 For $u \in \mathbb{N}$ let $f \in C^{u}\left(\mathbb{R}^{2}\right)$ and $f_{j}:=f \phi_{j}$. Then for $i \in\{\mathrm{h}, \mathrm{v}\}$ and any $\mathbf{r} \in \mathbb{N}_{0}^{2}$ we have

$$
\int_{\operatorname{supp} \Psi_{j, \ell}^{(i)}}\left|\partial^{\mathbf{r}}\left[\mathcal{F} f_{j}\right](\boldsymbol{\xi})\right|^{2} \mathrm{~d} \boldsymbol{\xi} \leq C(u, \mathbf{r}) 2^{-j\left(2 u+1+|\mathbf{r}|_{1}\right)} .
$$

Proof Since $\phi_{j} \in C_{0}^{\infty}\left(\mathbb{R}^{2}\right)$ we have $f_{j} \in C^{u}\left(\mathbb{R}^{2}\right)$ and using Eq. 17 we get

$$
\partial^{(u, 0)} f_{j}=\sum_{s=0}^{u}\left(\begin{array}{l}
u \\
s
\end{array}\right) \partial^{(s, 0)} \phi_{j} \partial^{(u-s, 0)} f=\sum_{s=0}^{u} \eta_{s},
$$

where $\eta_{s}:=\left(\begin{array}{l}u \\ s\end{array}\right) \partial^{(s, 0)} \phi_{j} \partial^{(u-s, 0)} f$. The function $\eta_{s}$ is $s$-times continuously differentiable with respect to the variable $\xi_{1}$. For $0 \leq t \leq s$, we can estimate:

$$
\left\|\partial^{(s+t, 0)} \phi_{j}\right\|_{\mathbb{R}^{2}, \infty}=\left\|2^{j(s+t) / 2} \frac{\partial^{s+t} \phi}{\partial \xi_{1}^{s+t}}\left(2^{j / 2} \cdot\right)\right\|_{\mathbb{R}^{2}, \infty} \leq C_{1} 2^{j(s+t) / 2} \leq C_{1} 2^{j s},
$$

which leads to

$$
\left\|\partial^{(s, 0)} \eta_{s}\right\|_{\mathbb{R}^{2}, \infty}=\left\|\left(\begin{array}{l}
u \\
s
\end{array}\right) \sum_{t=0}^{s}\left(\begin{array}{l}
s \\
t
\end{array}\right) \partial^{(s+t, 0)} \phi_{j} \partial^{(u-t, 0)} f\right\|_{\mathbb{R}^{2}, \infty} \leq C_{2}(u, s) 2^{j s} .
$$

By definition of the function $\phi_{j}$, we have $\left|\operatorname{supp} \phi_{j}\right| \leq 2^{-j}$ and with property Eq. 13 and the Plancherel theorem we get

$$
\int_{\mathbb{R}^{2}}\left|(2 \pi)\left(\mathrm{i} \xi_{1}\right)^{s} \mathcal{F} \eta_{s}(\boldsymbol{\xi})\right|^{2} \mathrm{~d} \boldsymbol{\xi}=\int_{\mathbb{R}^{2}}\left|\partial^{(s, 0)} \eta_{s}(\mathbf{x})\right|^{2} \mathrm{~d} \mathbf{x} \leq C_{2}(u) 2^{j(2 s-1)} .
$$

For the first variable in supp $\Psi_{j, \ell}^{(i)}$ we have $2^{j-1} \leq \xi_{1} \leq 2^{j+1}$ leading to

$$
\begin{aligned}
(2 \pi)^{2}\left(\mathrm{i} 2^{j-1}\right)^{2 s} \int_{W_{j, \ell}^{(i)}}\left|\mathcal{F} \eta_{s}(\boldsymbol{\xi})\right|^{2} \mathrm{~d} \boldsymbol{\xi} & \leq \int_{W_{j, \ell}^{(i)}}\left|(2 \pi)\left(\mathrm{i} \xi_{1}\right)^{s} \mathcal{F} \eta_{s}(\boldsymbol{\xi})\right|^{2} \mathrm{~d} \boldsymbol{\xi} \\
& \leq C_{2}(u) 2^{j(2 s-1)}
\end{aligned}
$$

which implies

$$
\int_{W_{j, \ell}^{(i)}}\left|\mathcal{F} \eta_{s}(\xi)\right|^{2} \mathrm{~d} \xi \leq C_{3}(u) 2^{-j}
$$


for all $0 \leq s \leq u$. Using again Eq. 13, it follows that

$$
\left(\mathrm{i} \xi_{1}\right)^{u} \mathcal{F} f_{j}=\mathcal{F}\left[\partial^{(u, 0)} f_{j}\right]=\sum_{s=0}^{u} \mathcal{F} \eta_{s},
$$

which leads together with Eq. 19 to

$$
\begin{aligned}
\int_{W_{j, \ell}^{(i)}}\left|\mathcal{F} f_{j}(\xi)\right|^{2} \mathrm{~d} \xi & \leq C_{4}(u) 2^{-2 j u} \int_{W_{j, \ell}^{(i)}}\left|\left(\mathrm{i} \xi_{1}\right)^{u} \mathcal{F} f_{j}(\xi)\right|^{2} \mathrm{~d} \xi \\
& \leq C_{5}(u) 2^{-2 j u} \sum_{s=0}^{u} \int_{W_{j, \ell}^{(i)}}\left|\mathcal{F} \eta_{s}(\xi)\right|^{2} \mathrm{~d} \boldsymbol{\xi} \\
& \leq C_{6}(u) 2^{-j(2 u+1)} .
\end{aligned}
$$

Next, we consider the function

$$
\mathbf{x}^{\mathbf{r}} f_{j}(\mathbf{x})=2^{-j|\mathbf{r}|_{1} / 2} f(\mathbf{x}) 2^{j|\mathbf{r}|_{1} / 2} \mathbf{x}^{\mathbf{r}} \phi_{j}(\mathbf{x})=2^{-j|\mathbf{r}|_{1} / 2} f(\mathbf{x}) \phi_{\mathbf{r}}\left(2^{j / 2} \mathbf{x}\right),
$$

where $\phi_{\mathbf{r}}(\mathbf{x}):=\mathbf{x}^{\mathbf{r}} \phi_{j}(\mathbf{x})$ and see that $\phi_{\mathbf{r}}\left(2^{j / 2} \cdot\right) \in C_{0}^{\infty}\left(\mathbb{R}^{2}\right)$ with $\left|\operatorname{supp} \phi_{\mathbf{r}}\right| \leq 2^{-j}$ is fulfilled. Thus, the Fourier transform of the function $f(\mathbf{x}) \phi_{\mathbf{r}}\left(2^{j / 2} \mathbf{x}\right)$ satisfies Eq. 20 with a constant $C_{6}(u, \mathbf{r})$. We use Eq. 12 to deduce

$$
\partial^{\mathbf{r}} \mathcal{F} f_{j}(\boldsymbol{\xi})=\mathcal{F}\left[(\mathrm{i} \mathbf{x})^{\mathbf{r}} f_{j}(\mathbf{x})\right](\boldsymbol{\xi})=\mathrm{i}^{\mathbf{r}} 2^{-j|\mathbf{r}|_{1} / 2} \mathcal{F}\left[f(\mathbf{x}) \phi_{\mathbf{r}}\left(2^{j / 2} \mathbf{x}\right)\right](\boldsymbol{\xi}),
$$

which leads to

$$
\begin{aligned}
\int_{W_{j, \ell}^{(i)}}\left|\partial^{\mathbf{r}} \mathcal{F} f_{j}(\boldsymbol{\xi})\right|^{2} \mathrm{~d} \boldsymbol{\xi} & =2^{-j|\mathbf{r}|_{1}} \int_{W_{j, \ell}^{(i)}}\left|\mathcal{F}\left[f(\mathbf{x}) \phi_{\mathbf{r}}\left(2^{j / 2} \mathbf{x}\right)\right](\boldsymbol{\xi})\right|^{2} \mathrm{~d} \boldsymbol{\xi} \\
& \leq C_{7}(u, \mathbf{r}) 2^{-j\left(2 u+1+|\mathbf{r}|_{1}\right)} .
\end{aligned}
$$

Lemma 4 For $u \in \mathbb{N}$ let $f \in C^{u}\left(\mathbb{R}^{2}\right)$ and $f_{j}:=f \phi_{j}$. Moreover, for $i \in\{\mathrm{h}, \mathrm{v}\}$ and $q \geq$ 2 let $\Psi^{(i)} \in \mathcal{W}_{2}^{q}$ be given. Then for $Q \in \mathcal{Q}_{j}^{0}$ and any $\mathbf{r} \in \mathbb{N}_{0}^{2}$ with $|\mathbf{r}|_{1} \leq q$ we have

$$
\left\|\partial^{\mathbf{r}}\left[\mathcal{F}\left[f_{j}\right] \Psi_{j, \ell}^{(i)}\right]\right\|_{\mathbb{R}^{2}, 2}^{2} \leq C(u, q) 2^{-j\left(2 u+1+|\mathbf{r}|_{1}\right)} .
$$

Proof For the partial derivative of the product inside of the norm, we use the multivariate Leibniz rule Eq. 17 and obtain

$$
\left\|\partial^{\mathbf{r}}\left[\mathcal{F}\left[f_{j}\right] \Psi_{j, \ell}^{(i)}\right]\right\|_{\mathbb{R}^{2}, 2}^{2} \leq \sum_{\mathbf{0} \leq \mathbf{s} \leq \mathbf{r}}\left(\begin{array}{l}
\mathbf{r} \\
\mathbf{s}
\end{array}\right) \int_{\mathbb{R}^{2}}\left|\partial^{\mathbf{s}}\left[\mathcal{F} f_{Q}\right](\boldsymbol{\xi}) \partial^{\mathbf{r}-\mathbf{s}}\left[\Psi_{j, \ell}^{(i)}\right](\boldsymbol{\xi})\right|^{2} \mathrm{~d} \boldsymbol{\xi} .
$$

Lemma 2 implies that for all $\xi \in \mathbb{R}^{2}$ the inequality

$$
\left|\partial^{\mathbf{r}-\mathbf{s}}\left[\Psi_{j, \ell}^{(i)}\right](\boldsymbol{\xi})\right|^{2} \leq C_{1}(q) 2^{-j\left(|\mathbf{r}|_{1}-|\mathbf{s}|_{1}\right)}
$$


holds, independent of the orientation parameter $\ell$. Together with Lemma 3, we have

$$
\begin{aligned}
\left\|\partial^{\mathbf{r}}\left(\mathcal{F}\left[f_{j}\right] \Psi_{j, \ell}^{(i)}\right)\right\|_{\mathbb{R}^{2}, 2}^{2} & \leq \sum_{\mathbf{0} \leq \mathbf{s} \leq \mathbf{r}}\left(\begin{array}{l}
\mathbf{r} \\
\mathbf{s}
\end{array}\right) \sup _{\boldsymbol{\xi} \in \mathbb{R}^{2}}\left|\partial^{\mathbf{r}-\mathbf{s}}\left[\Psi_{j, \ell}^{(i)}\right](\boldsymbol{\xi})\right|^{2} \\
& \times \int_{\operatorname{supp} \Psi_{j, \ell}^{(i)}}\left|\partial^{\mathbf{s}}\left[\mathcal{F} f_{j}\right](\boldsymbol{\xi})\right|^{2} \mathrm{~d} \boldsymbol{\xi} \\
& \leq \sum_{\mathbf{0} \leq \mathbf{s} \leq \mathbf{r}}\left(\begin{array}{l}
\mathbf{r} \\
\mathbf{s}
\end{array}\right) C_{2}(u, q) 2^{-j\left(|\mathbf{r}|_{1}-|\mathbf{s}|_{1}\right)} 2^{-j\left(2 u+1+|\mathbf{s}|_{1}\right)} \\
& =C_{3}(u, q) 2^{-j\left(2 u+1+|\mathbf{r}|_{1}\right)} .
\end{aligned}
$$

Following the approach from [4, Chapter 6.1], we assume that for $j \geq j_{0}$ the edge curve $\partial T$ can be parametrized on the support of $\phi_{Q}, Q \in \mathcal{Q}_{j}^{1}$, either as $\left(x_{1}, E\left(x_{1}\right)\right)^{\mathrm{T}}$ or $\left(E\left(x_{2}\right), x_{2}\right)^{\mathrm{T}}$.

Definition 1 For $x_{2} \in\left[-2^{-j / 2}, 2^{-j / 2}\right]$ let $\left(E\left(x_{2}\right), x_{2}\right)^{\mathrm{T}}$ be a parametrization of $\partial T$ with $E(0)=E^{\prime}(0)=0$. For $f \in C^{2}\left(\mathbb{R}^{2}\right)$, we call:

$$
\mathcal{E}_{j}(\mathbf{x})=f(\mathbf{x}) \phi_{j}(\mathbf{x}) \chi_{\left\{x_{1} \geq E\left(x_{2}\right)\right\}}(\mathbf{x})
$$

standard edge fragment.

Let $\mathcal{E}_{j, \mathbf{x}_{0}, \gamma}$ be an arbitrary edge fragment, which tangent in the point $\mathbf{x}_{0} \in \partial T$ is pointing in the direction $(\cos \gamma, \sin \gamma)^{\mathrm{T}}$ for $\gamma \in[0,2 \pi)$. Clearly, $\mathcal{E}_{j, \mathbf{0}, 0}=\mathcal{E}_{j}$ is a standard edge fragment. Moreover, in [4, Corollary 6.7], it is remarked that, although an arbitrary edge fragment $\mathcal{E}_{j, \mathbf{x}_{0}, \gamma}$ can not be obtained via rotation and translation of a standard edge fragment, we have the connection:

$$
\mathcal{F} \mathcal{E}_{j, \mathbf{x}_{0}, \gamma}(\boldsymbol{\xi})=\mathrm{e}^{-\mathrm{i} \mathbf{x}_{0}^{\mathrm{T}} \boldsymbol{\xi}} \mathcal{F} \mathcal{E}_{j}\left(\mathbf{R}_{\gamma}^{\mathrm{T}} \boldsymbol{\xi}\right)
$$

of their Fourier transforms. The following lemma is a consequence of [4, Corollary 6.6].

Lemma 5 For $j \in \mathbb{N}$ let $I_{j}=\left[2^{j-1}, 2^{j+1}\right]$ and $\mathcal{E}_{j}$ be a standard edge fragment. Then for angles $\theta, \gamma \in[0,2 \pi)$ and $\mathbf{r} \in \mathbb{N}_{0}^{2}$, we have:

$$
\int_{|\rho| \in I_{j}}\left|\partial^{\mathbf{r}}\left[\mathcal{F} \mathcal{E}_{j}\right](\rho \boldsymbol{\Theta}(\theta-\gamma))\right|^{2} \mathrm{~d} \rho \leq C(\mathbf{r}) 2^{-j\left(2+|\mathbf{r}|_{1}\right)}\left(1+2^{j / 2}|\sin (\theta-\gamma)|\right)^{-5} \text {. }
$$

We can deduce the following result, whose proof uses ideas from [12, Proposition 2.1].

Lemma 6 For $i \in\{\mathrm{h}, \mathrm{v}\}$ let $\Psi^{(i)} \in \mathcal{W}_{2}^{q}$. Then for a standard edge fragment $\mathcal{E}_{j}$, a rotation matrix $\mathbf{R}_{\gamma}$ by the angle $\gamma \in[0,2 \pi)$ and $\mathbf{r} \in \mathbb{N}_{0}^{2}$ we have

$$
\int_{\operatorname{supp} \Psi_{j, \ell}^{(i)}}\left|\partial^{\mathbf{r}}\left[\mathcal{F} \mathcal{E}_{j}\right]\left(\mathbf{R}_{\gamma}^{\mathrm{T}} \boldsymbol{\xi}\right)\right|^{2} \mathrm{~d} \boldsymbol{\xi} \leq C(\mathbf{r}) 2^{-j\left(3 / 2+|\mathbf{r}|_{1}\right)}\left(1+2^{j / 2}\left|\sin \left(\theta_{j, \ell}^{(i)}-\gamma\right)\right|\right)^{-5} .
$$


Proof From Lemma 1, we know supp $\Psi_{j, \ell}^{(i)} \subset W_{j, \ell}^{(i)}$ and we transform the integral into polar coordinates and use Lemma 5 to obtain:

$$
\begin{aligned}
\int_{\operatorname{supp} \Psi_{j, \ell}^{(i)}}\left|\partial^{\mathbf{r}}\left[\mathcal{F} \mathcal{E}_{j}\right]\left(\mathbf{R}_{\gamma}^{\mathrm{T}} \boldsymbol{\xi}\right)\right|^{2} \mathrm{~d} \boldsymbol{\xi} & \leq 2^{j+1} \int_{\theta_{j, \ell-2}^{(i)}}^{\theta_{j, \ell+2}^{(i)}} \int_{2^{j}}^{2^{j+1}}\left|\partial^{\mathbf{r}}\left[\mathcal{F} \mathcal{E}_{j}\right](\rho \boldsymbol{\Theta}(\theta-\gamma))\right|^{2} \mathrm{~d} \rho \mathrm{d} \theta \\
& \leq C(\mathbf{r}) 2^{-j\left(1+|\mathbf{r}|_{1}\right)} \int_{\theta_{j, \ell-2}^{(i)}}^{\theta_{j, \ell+2}^{(i)}}\left(1+2^{j / 2}|\sin (\theta-\gamma)|\right)^{-5} \mathrm{~d} \theta \\
& \leq C_{2}(\mathbf{r}) 2^{-j\left(3 / 2+|\mathbf{r}|_{1}\right)}\left(1+2^{j / 2}\left|\sin \left(\theta_{j, \ell}^{(i)}-\gamma\right)\right|\right)^{-5} .
\end{aligned}
$$

Lemma 7 For $i \in\{\mathrm{h}, \mathrm{v}\}$ let $\Psi^{(i)} \in \mathcal{W}_{2}^{q}$. Then for a standard edge fragment $\mathcal{E}_{j}, a$ rotation matrix $\mathbf{R}_{\gamma}$ by the angle $\gamma \in[0,2 \pi)$ and $\mathbf{r} \in \mathbb{N}_{0}^{2}$ we have

$$
\left\|\partial^{\mathbf{r}}\left[\mathcal{F} \mathcal{E}_{j}\left(\mathbf{R}_{\gamma}^{\mathrm{T}} \cdot\right) \Psi_{j, \ell}^{(i)}\right]\right\|_{\mathbb{R}^{2}, 2}^{2} \leq C(q) 2^{-j\left(3 / 2+|\mathbf{r}|_{1}\right)}\left(1+2^{j / 2}\left|\sin \left(\theta_{j, \ell}^{(i)}-\gamma\right)\right|\right)^{-5} .
$$

Proof We repeat the steps of the proof of Lemma 4 and use Lemma 6 instead of Lemma 3 in the last step.

The Laplace operator is denoted by $\Delta:=\partial^{(2,0)}+\partial^{(0,2)}$ and for $q \in \mathbb{N}_{0}$ we have

$$
\Delta^{q}=\sum_{|\mathbf{r}|_{1}=q}\left(\begin{array}{l}
q \\
\mathbf{r}
\end{array}\right) \partial^{2 \mathbf{r}}
$$

For the next lemma, we define the second-order differential operator $L:=I+2^{j} \Delta$, which was already used in $[4,12]$. Using Eq. 22, we have:

$$
L^{q}=\left(I+2^{j} \Delta\right)^{q}=\sum_{s=0}^{q}\left(\begin{array}{l}
q \\
s
\end{array}\right) 2^{j s} \Delta^{s}=\sum_{s=0}^{q}\left(\begin{array}{l}
q \\
s
\end{array}\right) 2^{j s} \sum_{|\mathbf{r}|_{1}=s}\left(\begin{array}{l}
s \\
\mathbf{r}
\end{array}\right) \partial^{2 \mathbf{r}} .
$$

Lemma 8 For $u \in \mathbb{N}$ let $f \in C^{u}\left(\mathbb{R}^{2}\right)$ and $f_{j}:=f \phi_{j}$. Moreover for $i \in\{\mathrm{h}, \mathrm{v}\}$ let $\Psi^{(i)} \in \mathcal{W}_{2}^{2 q}$ with $q \geq 2$. Then we have

$$
\left\|L^{q}\left[\mathcal{F}[h] \Psi_{j, \ell}^{(i)}\right]\right\|_{\mathbb{R}^{2}, 2}^{2} \leq \begin{cases}C_{1}(u, q) 2^{-j(2 u+1)}, & h=f_{j}, \\ C_{2}(q) 2^{-3 j / 2}\left(1+2^{j / 2}\left|\sin \left(\theta_{j, \ell}^{(i)}-\gamma\right)\right|\right)^{-5} & h=\mathcal{E}_{j}\left(\mathbf{R}_{\gamma}^{\mathrm{T}} \cdot\right) .\end{cases}
$$


Proof By applying the Cauchy-Schwarz inequality twice, we obtain

$$
\left\|L^{q}\left[\mathcal{F}[h] \Psi_{j, \ell}^{(i)}\right]\right\|_{\mathbb{R}^{2}, 2}^{2} \leq q \sum_{s=0}^{q}\left(\begin{array}{l}
q \\
s
\end{array}\right)^{2}(s+1) 2^{2 j s} \sum_{|\mathbf{r}|_{1}=s}\left(\begin{array}{l}
s \\
\mathbf{r}
\end{array}\right)^{2}\left\|\partial^{2 \mathbf{r}}\left[\mathcal{F}[h] \Psi_{j, \ell}^{(i)}\right]\right\|_{\mathbb{R}^{2}, 2}^{2}
$$

and get the result by inserting the corresponding upper bounds for the norm from Lemma 4 and Lemma 7.

The last part of this section consists of lemmata which are needed to proof Theorem 2. We start with some important localization properties.

Lemma 9 For $i \in\{\mathrm{h}, \mathrm{v}\}$ and $q \geq 2$ let $\Psi^{(i)} \in \mathcal{W}_{2}^{2 q}$ be given. Then for all $\mathbf{x} \in$ $[-\pi, \pi)^{2}$ we have

$$
\left|\psi_{j, \ell, \mathbf{y}}^{(i)}(\mathbf{x})\right| \leq C(q) 2^{3 j / 2} \min \left\{1, \frac{\left(1+2^{(j+1) / 2}\left|\sin \left(\theta_{j, \ell}^{(i)}-\gamma\right)\right|\right)^{q}}{\left(2^{j}|\mathbf{x}-2 \pi \widetilde{\mathbf{y}}|_{2}\right)^{q}}\right\}
$$

where $\mathbf{x}-2 \pi \tilde{\mathbf{y}}=|\mathbf{x}-2 \pi \tilde{\mathbf{y}}|_{2}(\cos \gamma, \sin \gamma)^{\mathrm{T}}$ for $\gamma \in[0,2 \pi)$.

Proof The function $\Psi_{j, \ell}^{(\mathrm{h})}$ is nonnegative leading to

$$
\left|\psi_{j, \ell, \mathbf{y}}^{(\mathrm{h})}(\mathbf{x})\right| \leq \sum_{\mathbf{k} \in \mathbb{Z}^{2}} \Psi_{j, \ell}^{(\mathrm{h})}(\mathbf{k})=\psi_{j, \ell, \mathbf{y}}^{(\mathrm{h})}(2 \pi \widetilde{\mathbf{y}}) \leq C_{1} 2^{3 j / 2},
$$

where the last estimate follows from Eq. 7.

Since $\Psi_{j, \ell}^{(\mathrm{h})} \in \mathcal{W}_{2}^{2 q}$, we can use the Poisson summation formula Eq. 16 to arrive at

$$
\begin{aligned}
\left|\psi_{j, \ell, \mathbf{y}}^{(\mathrm{h})}(\mathbf{x})\right| & =\left|\sum_{\mathbf{k} \in \mathbb{Z}^{2}} \Psi_{j, \ell}^{(\mathrm{h})}(\mathbf{k}) \mathrm{e}^{\mathrm{i} \mathbf{k}^{\mathrm{T}}(\mathbf{x}-2 \pi \widetilde{\mathbf{y}})}\right| \\
& =\left|\sum_{\mathbf{n} \in \mathbb{Z}^{2}} \mathcal{F}^{-1} \Psi_{j, \ell}^{(\mathrm{h})}(\mathbf{x}-2 \pi(\widetilde{\mathbf{y}}-\mathbf{n}))\right| \leq \sum_{\mathbf{n} \in \mathbb{Z}^{2}}|S(\mathbf{n})|,
\end{aligned}
$$

where

$$
S(\mathbf{n}):=\int_{\mathbb{R}^{2}} \Psi_{j, \ell}^{(\mathrm{h})}(\boldsymbol{\xi}) \mathrm{e}^{\mathrm{i} \xi^{\mathrm{T}}(\mathbf{x}-2 \pi(\widetilde{\mathbf{y}}-\mathbf{n}))} \mathrm{d} \boldsymbol{\xi}
$$

Let $\mathbf{R}_{\gamma}$ be a rotation matrix by the angle $\gamma$. Then

$$
\mathbf{R}_{\gamma}^{\mathrm{T}}(\mathbf{x}-2 \pi \widetilde{\mathbf{y}})=|\mathbf{x}-2 \pi \widetilde{\mathbf{y}}|_{2} \mathbf{R}_{\gamma}^{\mathrm{T}}(\cos \gamma, \sin \gamma)^{\mathrm{T}}=|\mathbf{x}-2 \pi \widetilde{\mathbf{y}}|_{2}(1,0)^{\mathrm{T}}
$$

and in the integral $S(\mathbf{0})$ we use this rotation matrix for a change of variable to see

$$
S(\mathbf{0})=\int_{\mathbb{R}^{2}} \Psi_{j, \ell}^{(\mathrm{h})}\left(\mathbf{R}_{\gamma} \boldsymbol{\xi}\right) \mathrm{e}^{\mathrm{i} \xi_{1}|\mathbf{x}-2 \pi \widetilde{\mathbf{y}}|_{2}} \mathrm{~d} \boldsymbol{\xi}
$$


Since the function $\Psi_{j, \ell}^{(\mathrm{h})}$ is compactly supported, we can use $q$-times partial integration together with Lemma 2 and Eq. 7 to deduce

$$
\begin{aligned}
|S(\mathbf{0})| & \leq \frac{\sup _{\boldsymbol{\xi} \in \mathbb{R}^{2}}\left|\partial^{(q, 0)} \Psi_{j, \ell}^{(\mathrm{h})}\left(\mathbf{R}_{\gamma} \cdot\right)\right|}{|\mathbf{x}-2 \pi \widetilde{\mathbf{y}}|_{2}^{q}} \int_{\operatorname{supp} \Psi_{j, \ell}^{(\mathrm{h})}\left(\mathbf{R}_{\gamma \cdot}\right)} \mathrm{d} \boldsymbol{\xi} \\
& \leq \frac{C(q) 2^{3 j / 2}\left(1+2^{(j+1) / 2}\left|\sin \left(\theta_{j, \ell}^{(\mathrm{h})}-\gamma\right)\right|\right)^{q}}{\left(2^{j}|\mathbf{x}-2 \pi \widetilde{\mathbf{y}}|_{2}\right)^{q}} .
\end{aligned}
$$

Using the same idea as before, we substitute with the rotation matrices $\mathbf{R}_{\gamma_{\mathbf{n}}}$ in the integrals $S(\mathbf{n})$, where $\gamma_{\mathbf{n}}$ is the direction of the vector $\mathbf{x}-2 \pi(\widetilde{\mathbf{y}}-\mathbf{n})$. Similar to Eq. 25 we use $2 q$-times integration by parts with respect to the first variable, Lemma 2 and Eq. 7 to obtain

$$
\begin{aligned}
|S(\mathbf{n})| & \leq \frac{C_{2}(q) 2^{3 j / 2}\left(1+2^{(j+1) / 2}\left|\sin \left(\theta_{j, \ell}^{(\mathrm{h})}-\gamma_{\mathbf{n}}\right)\right|\right)^{2 q}}{\left(2^{j}|\mathbf{x}-2 \pi(\tilde{\mathbf{y}}-\mathbf{n})|_{2}\right)^{2 q}} \\
& \leq \frac{C_{3}(q) 2^{3 j / 2}}{\left(2^{j / 2}|\mathbf{x}-2 \pi(\widetilde{\mathbf{y}}-\mathbf{n})|_{2}\right)^{2 q}}
\end{aligned}
$$

Observe that

$$
\pi^{2} \geq \pi|\mathbf{x}-2 \pi \widetilde{\mathbf{y}}|_{\infty} \geq \frac{\pi}{\sqrt{2}}|\mathbf{x}-2 \pi \widetilde{\mathbf{y}}|_{2} \geq|\mathbf{x}-2 \pi \widetilde{\mathbf{y}}|_{2}
$$

and with the inverse triangle inequality we can estimate

$$
|\mathbf{x}-2 \pi(\tilde{\mathbf{y}}-\mathbf{n})|_{2} \geq 2 \pi|\mathbf{n}|_{\infty}-|\mathbf{x}-2 \pi \widetilde{\mathbf{y}}|_{\infty} \geq \sqrt{|\mathbf{x}-2 \pi \widetilde{\mathbf{y}}|_{2}}\left(2|\mathbf{n}|_{\infty}-1\right) .
$$

Since $\left|\left\{\mathbf{n} \in \mathbb{Z}^{2} ;|\mathbf{n}|_{\infty}=k, k \in \mathbb{N}\right\}\right|=8 k$ we use Eq. 26 to conclude

$$
\begin{aligned}
\sum_{\mathbf{n} \in \mathbb{Z}^{2} \backslash\{\mathbf{0}\}}|S(\mathbf{n})| & \leq C_{3}(q) 2^{-j q} 2^{3 j / 2} \sum_{\mathbf{n} \in \mathbb{Z}^{2} \backslash\{\mathbf{0}\}}|\mathbf{x}-2 \pi(\widetilde{\mathbf{y}}-\mathbf{n})|_{2}^{-2 q} \\
& \leq \frac{C_{3}(q) 2^{3 j / 2}}{\left(2^{j}|\mathbf{x}-2 \pi \widetilde{\mathbf{y}}|_{2}\right)^{q}} \sum_{k=1}^{\infty} \frac{8 k}{(2 k-1)^{2 q}}
\end{aligned}
$$

and the infinite sum in the last line converges because $q \geq 2$. We finish the proof by making use of Eqs. 25 and 27 in Eq. 24.

Let $\gamma:[0,2 \pi) \rightarrow \partial T$ be a parametrization of the boundary $\partial T$. We assume there is $M \in \mathbb{N}$ such that for each $x \in\left[a_{k}, b_{k}\right], k=1, \ldots, M$, the curve $\gamma$ can either be represented as a horizontal curve $(x, f(x))^{\mathrm{T}}$ or a vertical curve $(f(x), x)^{\mathrm{T}}$. Depending on the choice of the parameter $i \in\{\mathrm{h}, \mathrm{v}\}$, we will distinguish if a curve is horizontal or vertical. If $i=\mathrm{h}$ then $(f(x), x)^{\mathrm{T}}$ with $\left|f^{\prime}(x)\right| \leq 1$ is a vertical curve and $(x, f(x))^{\mathrm{T}}$ with $\left|f^{\prime}(x)\right|<1$ is a horizontal curve. On the other hand, if $i=\mathrm{v}$ then $(f(x), x)^{\mathrm{T}}$ with $\left|f^{\prime}(x)\right|<1$ is a vertical curve and $(x, f(x))^{\mathrm{T}}$ with $\left|f^{\prime}(x)\right| \leq 1$ is a horizontal curve. 
Let $\mathbf{y}=\left(y_{1}, y_{2}\right)^{\mathrm{T}} \in \mathcal{P}\left(\mathbf{N}_{j, \ell}^{(\mathrm{h})}\right)$. We assume that the boundary curve can be vertically parametrized by $(f(x), x)^{\mathrm{T}}$ for $\left|x-2 \pi y_{2}\right| \leq 2^{-j / 2}$. For $m:=f^{\prime}\left(2 \pi y_{2}\right) \in$ $[-1,1]$ and $A:=\frac{1}{2} f^{\prime \prime}\left(2 \pi y_{2}\right)$ let

$$
T_{\mathbf{y}}(x)=f\left(2 \pi y_{2}\right)+m\left(x-2 \pi y_{2}\right)+A\left(x-2 \pi y_{2}\right)^{2}
$$

be the second-order Taylor approximation for $f(x)$ in the point $x_{0}=2 \pi y_{2}$. Denote by $\widehat{\mathcal{T}}_{\mathbf{y}}^{(\mathrm{h})}$ the modified version of $\mathcal{T}$ by replacing the function $f(x)$ by the approximation $T_{\mathbf{y}}(x)$ for $\left|x-2 \pi y_{2}\right| \leq 2^{-j / 2}$ if the corresponding parametrization is a vertical curve and similarly $\widehat{\mathcal{T}}_{\mathbf{y}}^{(\mathrm{v})}$ as the modified version of $\mathcal{T}$ if the parametrization is a horizontal curve. Although this notation seems to be counterintuitive, it is convenient since by Lemma 11 only the interaction of horizontal wavelets with vertical curves and vertical wavelets with horizontal curves contributes to the desired lower bound in Theorem 2. The analog of the following lemma can be found in [17] for the discrete and in $[13,14]$ for the continuous setting.

Lemma 10 For $i \in\{\mathrm{h}, \mathrm{v}\}$ and large $q \in \mathbb{N}$ let $\Psi^{(i)} \in \mathcal{W}_{2}^{2 q}$ be given. Then for $\mathbf{y} \in \mathcal{P}\left(\mathbf{N}_{j, \ell}^{(i)}\right)$ we have

$$
\left|\left\langle\mathcal{T}^{2 \pi}-\left(\widehat{\mathcal{T}}_{\mathbf{y}}^{(i)}\right)^{2 \pi}, \psi_{j, \ell, \mathbf{y}}^{(i)}\right\rangle_{2}\right| \leq C(q) 2^{-j / 4}
$$

Proof We only show the proof for $i=\mathrm{h}$ since the other case is similar. For this proof, we define the set $B_{j}=\left\{\left(x_{1}, x_{2}\right)^{\mathrm{T}} \in[-\pi, \pi)^{2}:\left|x_{2}-2 \pi \tilde{y}_{2}\right| \leq 2^{-7 j / 16}\right\}$ and write

$$
\begin{aligned}
\left|\left\langle\mathcal{T}^{2 \pi}-\left(\widehat{\mathcal{T}}_{\mathbf{y}}^{(\mathrm{h})}\right)^{2 \pi}, \psi_{j, \ell, \mathbf{y}}^{(\mathrm{h})}\right\rangle_{2}\right| & \leq \int_{\mathbb{T}^{2}}\left|\psi_{j, \ell, \mathbf{y}}^{(\mathrm{h})}(\mathbf{x})\right|\left|\chi_{T}(\mathbf{x})-\chi_{\widehat{T}_{\mathbf{y}}^{(\mathrm{h})}}(\mathbf{x})\right| \mathrm{d} \mathbf{x} \\
& =\left(\int_{B_{j}}+\int_{B_{j}^{\mathrm{c}}}\right)\left|\psi_{j, \ell, \mathbf{y}}^{(\mathrm{h})}(\mathbf{x})\right| \mid \chi_{T}(\mathbf{x})-\chi_{\widehat{T}_{\mathbf{y}}^{(\mathrm{h})}(\mathbf{x}) \mid \mathrm{d} \mathbf{x}} \\
& =: \mathcal{I}_{1}+\mathcal{I}_{2} .
\end{aligned}
$$

Using the definition of $T_{\mathbf{y}}(x)$ in Eq. 28, we can estimate

$$
\left|f(x)-T_{\mathbf{y}}(x)\right| \leq C\left|x-2 \pi y_{2}\right|^{3}
$$

for the area between $\mathcal{T}$ and $\widehat{\mathcal{T}}_{\mathbf{y}}^{(\mathrm{h})}$ if $\left|x-2 \pi y_{2}\right| \leq 2^{-j / 2}$. From Lemma 9 , we can obtain the uniform bound $\left|\psi_{j, \ell, \mathbf{y}}^{(\mathrm{h})}(\mathbf{x})\right| \leq C(q) 2^{3 j / 2}$ and we can estimate the first integral by

$$
\left|\mathcal{I}_{1}\right| \leq C(q) 2^{3 j / 2} \int_{\left|x-2 \pi \tilde{y}_{2}\right| \leq 2^{-7 j / 16}}\left|x-2 \pi y_{2}\right|^{3} \mathrm{~d} x \leq C(q) 2^{3 j / 2} 2^{-7 j / 4}=C(q) 2^{-j / 4} .
$$


In addition, we use again Lemma 9 but this time for the decay term in the minimum to arrive at

$$
\begin{aligned}
\left|\mathcal{I}_{2}\right| & \leq C(q) 2^{3 j / 2} \int_{\left|x-2 \pi \tilde{y}_{2}\right|>2^{-7 j / 16}}\left(2^{j / 2}\left|x-2 \pi \tilde{y}_{2}\right|\right)^{-q} \mathrm{~d} x \\
& \leq C_{3}(q) 2^{3 j / 2} 2^{-j q / 2} 2^{7(q-1) j / 16}=C_{3}(q) 2^{-j(q / 16-17 / 16)}
\end{aligned}
$$

for the second integral, which shows that the lemma is proved for $q \geq 21$.

From the divergence theorem, one can see that the Fourier transform of a characteristic function $\mathcal{T}=\chi_{T}$ is given by

$$
\mathcal{F} \mathcal{T}(\boldsymbol{\xi})=(2 \pi)^{-2} \int_{\mathbb{R}^{2}} \chi_{T}(\mathbf{x}) \mathrm{e}^{-\mathrm{i} \mathbf{x}^{\mathrm{T}} \boldsymbol{\xi}} \mathrm{d} \mathbf{x}=\frac{\mathrm{i}(2 \pi)^{-2}}{|\boldsymbol{\xi}|_{2}} \int_{\partial T} \mathrm{e}^{-\mathrm{i} \mathbf{x}^{\mathrm{T}} \boldsymbol{\xi}} \boldsymbol{\Theta}^{\mathrm{T}}(\theta) \mathbf{n}(\mathbf{x}) \mathrm{d} \sigma(\mathbf{x})
$$

with the outer normal vector $\mathbf{n}(\mathbf{x})$. We remind the parametrization of $\partial T$ given by $\boldsymbol{\gamma}(x), x \in[0,2 \pi)$ and use polar coordinates to represent the line integral Eq. 29 as

$$
\begin{aligned}
\mathcal{F} \mathcal{T}(\rho, \theta) & =\frac{\mathrm{i}}{(2 \pi)^{2} \rho} \int_{0}^{2 \pi} \mathrm{e}^{-\mathrm{i} \rho \boldsymbol{\Theta}^{\mathrm{T}}(\theta) \boldsymbol{\gamma}(x)} \boldsymbol{\Theta}^{\mathrm{T}}(\theta) \mathbf{n}(\boldsymbol{\gamma}(x))\left|\boldsymbol{\gamma}^{\prime}(x)\right|_{2} \mathrm{~d} x \\
& =\frac{\mathrm{i}}{(2 \pi)^{2} \rho} \sum_{k=1}^{M} \mathcal{I}_{k}(\rho, \theta),
\end{aligned}
$$

where

$$
\mathcal{I}_{k}(\rho, \theta):=\int_{a_{k}}^{b_{k}} \mathrm{e}^{-\mathrm{i} \rho \boldsymbol{\Theta}^{\mathrm{T}}(\theta) \boldsymbol{\gamma}_{k}(x)} \boldsymbol{\Theta}^{\mathrm{T}}(\theta) \boldsymbol{\beta}_{k}(x) \mathrm{d} x
$$

and $\boldsymbol{\beta}_{k}(x):=\mathbf{n}\left(\boldsymbol{\gamma}_{k}(x)\right) \sqrt{1+\left(f_{k}^{\prime}(x)\right)^{2}}$. Using this, we can conclude:

$$
\begin{aligned}
\mathcal{F}^{-1}\left[\mathcal{F}[\mathcal{T}] \Psi_{j, \ell}^{(i)}\right](2 \pi \widetilde{\mathbf{y}}) & =\int_{\mathbb{R}^{2}} \mathcal{F} \mathcal{T}(\boldsymbol{\xi}) \Psi_{j, \ell}^{(i)}(\boldsymbol{\xi}) \mathrm{e}^{2 \pi \mathrm{i} \xi} \widetilde{\mathbf{y}}_{\mathrm{d} \boldsymbol{\xi}} \\
& =\frac{\mathrm{i}}{(2 \pi)^{2}} \sum_{k=1}^{M} \int_{0}^{\infty} \int_{0}^{2 \pi} \Psi_{j, \ell}^{(i)}(\rho, \theta) \mathrm{e}^{2 \pi \mathrm{i} \rho \boldsymbol{\Theta}^{\mathrm{T}}(\theta) \widetilde{\mathbf{y}}} \mathcal{I}_{k}(\rho, \theta) \mathrm{d} \theta \mathrm{d} \rho \\
& =\frac{2^{j} \mathrm{i}}{(2 \pi)^{2}} \sum_{k=1}^{M} \int_{0}^{\infty} \int_{0}^{2 \pi} \Psi_{j, \ell}^{(i)}\left(2^{j} \rho, \theta\right) \mathrm{e}^{2 \pi \mathrm{i} 2^{j} \rho \boldsymbol{\Theta}^{\mathrm{T}}(\theta) \widetilde{\mathbf{y}}} \\
& \times \mathcal{I}_{k}\left(2^{j} \rho, \theta\right) \mathrm{d} \theta \mathrm{d} \rho,
\end{aligned}
$$

where we again transformed the integral into polar coordinates and the interchange of summation and integration is valid since the function $\Psi_{j, \ell}^{(i)}$ has finite support.

Denote by $\mathcal{M}^{(\mathrm{h})} \subset\{1, \ldots, M\}$ the set of all indices such that for $k \in \mathcal{M}^{(\mathrm{h})}$ the curve $\boldsymbol{\gamma}_{k}$ is horizontal and by $\mathcal{M}^{(\mathrm{v})} \subset\{1, \ldots, M\}$ the set of all indices such that for 
$k \in \mathcal{M}^{(\mathrm{v})}$ the curve $\boldsymbol{\gamma}_{k}$ is vertical. Obviously we have $\mathcal{M}^{(\mathrm{h})} \cup \mathcal{M}^{(\mathrm{v})}=\{1, \ldots, M\}$ and can prove the following lemma, whose idea of proof was given in [17].

Lemma 11 For $i \in\{\mathrm{h}, \mathrm{v}\}$ and $q \in \mathbb{N}$ let $\Psi^{(i)} \in \mathcal{W}_{2}^{q}$ be given. Then for any $n \in \mathbb{N}$ there is a constant $C(n)>0$ such that for every $k \in \mathcal{M}^{(i)}$ we have

$$
\left|\int_{0}^{\infty} \int_{0}^{2 \pi} \Psi_{j, \ell}^{(i)}\left(2^{j} \rho, \theta\right) \mathrm{e}^{2 \pi \mathrm{i} 2^{j} \rho \boldsymbol{\Theta}^{\mathrm{T}}(\theta) \widetilde{\mathbf{y}}} \mathcal{I}_{k}\left(2^{j} \rho, \theta\right) \mathrm{d} \theta \mathrm{d} \rho\right| \leq C(n) 2^{-j n} .
$$

Proof We show the result for $i=\mathrm{h}$ since the other case is similar. Suppose that $k \in \mathcal{M}^{(\mathrm{h})}$ and $\boldsymbol{\gamma}_{k}(x)=\left(x, f_{k}(x)\right)^{\mathrm{T}}$ for $x \in\left[a_{k}, b_{k}\right]$. Hence, the outer normal vector in $x$ is given by $\mathbf{n}\left(\boldsymbol{\gamma}_{k}(x)\right)=\left(f_{k}^{\prime}(x),-1\right)^{\mathrm{T}}$ leading to

$$
\mathcal{I}_{k}\left(2^{j} \rho, \theta\right)=\int_{a_{k}}^{b_{k}} \mathrm{e}^{-\mathrm{i} 2^{j} \rho \boldsymbol{\Theta}^{\mathrm{T}}(\theta)\left(x, f_{k}(x)\right)^{\mathrm{T}}}\left(f_{k}^{\prime}(x),-1\right) \boldsymbol{\Theta}(\theta) \beta_{k}(x) \mathrm{d} x .
$$

From the assumption on horizontal curves for $i=\mathrm{h}$ we have $\left|f_{k}^{\prime}(x)\right|<1$ and the support properties of the function $\Psi_{j, \ell}^{(\mathrm{h})}$ given in Lemma 1 imply

$$
|\theta| \leq \theta_{j, 2^{j / 2}+2}^{(\mathrm{h})}=\arctan \left(1+2^{1-j / 2}\right) \leq \frac{\pi}{4}+\delta
$$

for some small $\delta>0$. From that, we conclude:

$$
\begin{aligned}
\left|\frac{\partial}{\partial x}\left[\left(x, f_{k}(x)\right) \boldsymbol{\Theta}(\theta)\right]\right| & =\left|\frac{\partial}{\partial x}\left[\cos \theta\left(x+f_{k}(x) \tan \theta\right)\right]\right| \\
& \geq|\cos \theta|\left(1-\left|f_{k}^{\prime}(x) \tan \theta\right|\right) \geq C .
\end{aligned}
$$

For $n \in \mathbb{N}$, we do $n$-times integration by parts with respect to the variable $x$ and obtain $\left|\mathcal{I}_{k}\left(2^{j} \rho, \theta\right)\right| \leq 2^{-j N}$, which leads to

$$
\left|\int_{0}^{\infty} \int_{0}^{2 \pi} \Psi_{j, \ell}^{(\mathrm{h})}\left(2^{j} \rho, \theta\right) \mathrm{e}^{2 \pi \mathrm{i} 2^{j} \rho \boldsymbol{\Theta}^{\mathrm{T}}(\theta) \tilde{\mathbf{y}}} \mathcal{I}_{k}\left(2^{j} \rho, \theta\right) \mathrm{d} \theta \mathrm{d} \rho\right| \leq C(n) 2^{-j n}\left|\operatorname{supp} \Psi_{j, \ell}^{(\mathrm{h})}\left(2^{j} \cdot\right)\right| .
$$

Lemma 1 implies $\left|\operatorname{supp} \Psi_{j, \ell}^{(\mathrm{h})}\left(2^{j} \cdot\right)\right| \leq C$ and since $n \in \mathbb{N}$ was arbitrary the lemma is proven.

The proof of the following lemma was given in [14].

Lemma 12 For $i \in\{\mathrm{h}, \mathrm{v}\}$ and $q \in \mathbb{N}$ let $\Psi^{(i)} \in \mathcal{W}_{2}^{q}$ and $\mathbf{y}=\left(y_{1}, y_{2}\right)^{\mathrm{T}} \in \mathcal{P}\left(\mathbf{N}_{j, \ell}^{(i)}\right)$ be given. Then for any $n \in \mathbb{N}$ there is a constant $C(n)>0$ such that

$$
\begin{aligned}
& \left|\int_{0}^{\infty} \int_{0}^{2 \pi} \int_{\left|x-2 \pi y_{2}\right|>2^{-j / 2}} \Psi_{j, \ell}^{(i)}(\rho, \theta) \mathrm{e}^{\mathrm{i} 2{ }^{j} \rho \boldsymbol{\Theta}^{\mathrm{T}}(\theta)(2 \pi \tilde{\mathbf{y}}-\boldsymbol{\gamma}(x))} \boldsymbol{\Theta}^{\mathrm{T}}(\theta) \boldsymbol{\beta}(x) \mathrm{d} x \mathrm{~d} \theta \mathrm{d} \rho\right| \\
& \leq C(n) 2^{-j n} .
\end{aligned}
$$




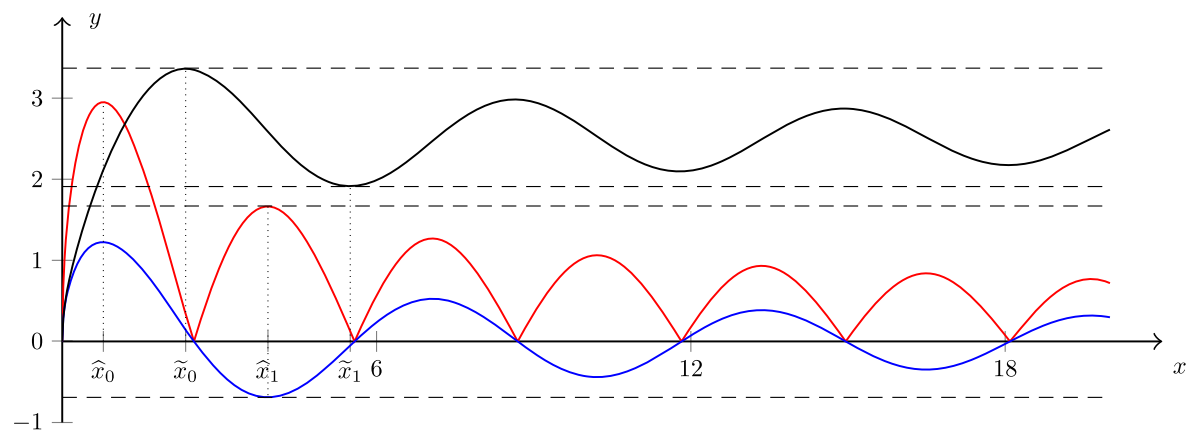

Fig. 6 Visualization of the functions $F^{+}(x)$ (black), $F^{-}(x)$ (blue) and $(1+\sqrt{2})\left|F^{-}(x)\right|$ (red) for $x \in$ $[0,20]$ together with upper and lower bounds of these functions (dashed lines) and the local extremal points from Lemma 14

The following lemma is a special case of [32, Proposition 8.3], called method of stationary phase.

Lemma 13 Let $\phi$ and $\varphi$ be smooth functions on the real line. Suppose $\phi^{\prime}\left(t_{0}\right)=0$ and $\phi^{\prime \prime}\left(t_{0}\right) \neq 0$. If $\varphi$ is supported in a sufficiently small neighborhood of $t_{0}$, then

$$
\int_{\mathbb{R}} \mathrm{e}^{\mathrm{i} \Lambda \phi(t)} \varphi(t) \mathrm{d} t=a_{0} \Lambda^{-1 / 2}+O\left(\Lambda^{-1}\right)
$$

as $\Lambda \rightarrow \infty$, where $a_{0}=\left(\frac{2 \pi \mathrm{i}}{\left|\phi^{\prime \prime}\left(t_{0}\right)\right|}\right)^{1 / 2} \varphi\left(t_{0}\right)$.

For $x \in(0, \infty)$, we introduce the so-called Fresnel integrals

$F c(x):=2 \int_{0}^{\sqrt{x}} \cos \left(v^{2}\right) \mathrm{d} v=\int_{0}^{x} \frac{\cos (v)}{\sqrt{v}} \mathrm{~d} v, \quad F s(x):=2 \int_{0}^{\sqrt{x}} \sin \left(t^{2}\right) \mathrm{d} t=\int_{0}^{x} \frac{\sin (v)}{\sqrt{v}} \mathrm{~d} v$

and define the functions $F^{+}(x):=F c(x)+F s(x)$ and $F^{-}(x):=F c(x)-F s(x)$ to show the following lemma (Fig. 6).

Lemma 14 We have

$$
\begin{array}{r}
F^{+}(x)>F^{-}(x)>0 \quad \text { for } 0<x<\frac{3 \pi}{4}, \\
F^{+}(x)>(1+\sqrt{2})\left|F^{-}(x)\right| \quad \text { for } \quad x \geq \frac{3 \pi}{4} .
\end{array}
$$

Proof It is clear that $F c(0)=F s(0)=0$ and for $x>0$ it is well known that $F c(x)$ $>0$ and $F s(x)>0$, which implies

$$
F^{+}(x)>F c(x)>F^{-}(x) .
$$


Moreover, we have

$$
\frac{\mathrm{d}}{\mathrm{d} x} F^{+}(x)=\frac{\cos x+\sin x}{\sqrt{x}}=0
$$

for $\tilde{x}_{k}:=\frac{3 \pi}{4}+k \pi, k \in \mathbb{N}_{0}$ and

$$
\frac{\mathrm{d}^{2}}{\mathrm{~d} x^{2}} F^{+}(x)=\frac{\cos x-\sin x}{\sqrt{x}}-\frac{\cos x+\sin x}{2(\sqrt{x})^{3}} .
$$

Since

$$
\frac{\cos \tilde{x}_{k}-\sin \tilde{x}_{k}}{\sqrt{\widetilde{x}_{k}}}-\frac{\cos \tilde{x}_{k}+\sin \tilde{x}_{k}}{2\left(\sqrt{\tilde{x}_{k}}\right)^{3}}=\frac{\cos \tilde{x}_{k}-\sin \tilde{x}_{k}}{\sqrt{\widetilde{x}_{k}}}= \begin{cases}-\sqrt{\frac{2}{\tilde{x}_{k}}}<0 & \text { for } k \text { even }, \\ \sqrt{\frac{2}{\widetilde{x}_{k}}}>0 & \text { for } k \text { odd },\end{cases}
$$

we see that $\tilde{x}_{k}$ is a local maximum point of $F^{+}$for $k$ even and a local minimum point for $k$ odd. To get information about global extremal points, we define

$$
I_{0}:=\int_{0}^{\tilde{x}_{0}} \frac{\cos v+\sin v}{\sqrt{v}} \mathrm{~d} v \quad \text { and } \quad I_{k}:=\int_{\tilde{x}_{k-1}}^{\tilde{x}_{k}} \frac{\cos v+\sin v}{\sqrt{v}} \mathrm{~d} v \text { for } k \geq 1
$$

and write $F^{+}\left(\tilde{x}_{k}\right)=\sum_{j=0}^{k} I_{j}$. For $v \in\left(\tilde{x}_{k-1}, \tilde{x}_{k}\right)$ we have $(\cos v+\sin v)>0$ for $k$ even and $(\cos v+\sin v)<0$ for $k$ odd. It follows that $I_{k}>0$ for $k$ even and $I_{k}<0$ for $k$ odd. Additionally, we have $I_{0}>3.36>1.45>\left|I_{1}\right|$ and since $v^{-1 / 2}$ is monotonically decreasing we have $\left|I_{k}\right|>\left|I_{k+1}\right|$ for $k \geq 1$.

Putting these observations together, we obtain $\left(I_{k}+I_{k+1}\right)>0$ for $k$ even and $\left(I_{k}+I_{k+1}\right)<0$ for $k$ odd. From that, we deduce that $\tilde{x}_{0}=\frac{3 \pi}{4}$ is the global maximum point of $F^{+}$since for even $k>0$ we have

$$
F^{+}\left(\tilde{x}_{k}\right)=I_{0}+\sum_{j=1}^{k} I_{j}<I_{0}=F^{+}\left(\tilde{x}_{0}\right)<3.37 \text {. }
$$

Similarly for $k>1$ odd, we can write

$$
F^{+}\left(\tilde{x}_{k}\right)=I_{0}+I_{1}+\sum_{j=2}^{k} I_{j}>I_{0}+I_{1}=F^{+}\left(\tilde{x}_{1}\right)>1.91,
$$

which shows that smallest local minimum is obtained at the point $\tilde{x}_{1}=\frac{7 \pi}{4}$ and thus $1.91<F^{+}(x)<3.37$ for $x \geq \tilde{x}_{0}$. For $0 \leq x<\tilde{x}_{0}$ we clearly have $0 \leq F^{+}(x)<$ 3.37 .

Similarly, one can show that $\widehat{x}_{k}:=\frac{\pi}{4}+k \pi, k \in \mathbb{N}_{0}$, is a local maximum point of $F^{-}$for $k$ even and a local minimum point for $k$ odd. Since $\widehat{x}_{0}<\frac{3 \pi}{4}<\widehat{x}_{1}=\frac{5 \pi}{4}$ and $F^{-}\left(\frac{3 \pi}{4}\right)>0.14>0$ we have $F^{-}(x)>0$ for $0<x<\frac{3 \pi}{4}$, which together with Eq. 32 gives the first statement of the lemma.

With similar arguments as in Eqs. 33 and 34, we have

$$
-0.69<F^{-}\left(\widehat{x}_{1}\right) \leq F^{-}(x) \leq F^{-}\left(\widehat{x}_{2}\right)<0.53
$$


for $x \geq \frac{3 \pi}{4}$. Since $(1+\sqrt{2}) 0.69<1.91$ we obtain $F^{+}(x)>(1+\sqrt{2})\left|F^{-}(x)\right|$ for $x \geq \frac{3 \pi}{4}$ and the proof is complete.

For the next lemma, we define the integrals

$$
\begin{aligned}
& a(\lambda, p, A):=\int_{0}^{\infty}(g(2 \sqrt{A \lambda v}+p \lambda)+g(2 \sqrt{A \lambda v}-p \lambda)) \frac{\cos v}{\sqrt{v}} \mathrm{~d} v, \\
& b(\lambda, p, A):=\int_{0}^{\infty}(g(2 \sqrt{A \lambda v}+p \lambda)+g(2 \sqrt{A \lambda v}-p \lambda)) \frac{\sin v}{\sqrt{v}} \mathrm{~d} v .
\end{aligned}
$$

Lemma 15 For $\lambda \in\left[\frac{1}{3}, \frac{4}{3}\right], p \in\left[-\frac{1}{4}, \frac{1}{4}\right]$ and $A>0$ we have $a(\lambda, p, A)>0$ and $b(\lambda, p, A)>0$ and at least one of the inequalities

$$
a(\lambda, p, A)-b(\lambda, p, A)>0
$$

or

$$
a(\lambda, p, A)+b(\lambda, p, A)>(1+\sqrt{2})|a(\lambda, p, A)-b(\lambda, p, A)|
$$

is true.

Proof We consider only the case $p \in\left[0, \frac{1}{4}\right]$ because $a(\lambda, p, A)$ and $b(\lambda, p, A)$ are symmetric in that variable. Let the function $h^{ \pm}:\left[\frac{1}{3}, \frac{4}{3}\right] \times\left[0, \frac{1}{4}\right] \times(0, \infty) \times[0, \infty) \rightarrow$ $\mathbb{R}$ be given by

$$
h^{ \pm}(\lambda, p, A, v):=2 \sqrt{A \lambda v} \pm p \lambda
$$

For fixed $(\lambda, p, A) \in\left[\frac{1}{3}, \frac{4}{3}\right] \times\left[0, \frac{1}{4}\right] \times(0, \infty)$, we have

$$
-\frac{1}{3} \leq-p \lambda=h^{-}(\lambda, p, A, 0) \leq h^{+}(\lambda, p, A, 0)=p \lambda \leq \frac{1}{3}
$$

and the functions $h^{ \pm}$are monotonically increasing in the variable $v \geq 0$. Since $g \in \mathcal{W}^{q}$ this implies that in the variable $v$ the functions $g\left(h^{+}(\lambda, p, A, v)\right)$ and $g\left(h^{-}(\lambda, p, A, v)\right)$ and hence the sum $\left(g\left(h^{+}(\lambda, p, A, v)\right)+g\left(h^{-}(\lambda, p, A, v)\right)\right)$ are also monotonically decreasing. Similarly, we have

$$
\begin{aligned}
h^{+}(\lambda, p, A, v) & \geq h^{-}(\lambda, p, A, v) \geq \frac{2}{3} \quad \text { if } \\
v & \geq \frac{p}{3 A}+\frac{1}{9 A \lambda}+\frac{p^{2} \lambda}{4 A}=: r(\lambda, p, A)>0,
\end{aligned}
$$

which leads to $\left(g\left(h^{+}(\lambda, p, A, v)\right)+g\left(h^{-}(\lambda, p, A, v)\right)\right)=0$ for $v \geq r(\lambda, p, A)$. Moreover, Eq. 37 implies

$$
\lim _{v \rightarrow 0^{+}} g\left(h^{+}(\lambda, p, A, v)\right)+g\left(h^{-}(\lambda, p, A, v)\right)=2
$$


and we use the mean value theorem of integration to deduce that there exists $x \in$ $(0, r(\lambda, p, A)]$ such that

$$
\begin{aligned}
a(\lambda, p, A) \pm b(\lambda, p, A)= & \int_{0}^{r(\lambda, p, A)}(g(2 \sqrt{A \lambda v}+p \lambda) \\
& +g(2 \sqrt{A \lambda v}-p \lambda)) \frac{\cos v \pm \sin v}{\sqrt{v}} \mathrm{~d} v \\
= & 2 F^{ \pm}(x) .
\end{aligned}
$$

Using Lemma 14, the proof is finished.

For the last lemma of this section, we define the following integrals:

$$
\begin{array}{r}
P_{1}(D, p, A):=\int_{\frac{1}{3}}^{\frac{4}{3}} \widetilde{g}(\lambda) \lambda^{-1}([a(\lambda, p, A)+b(\lambda, p, A)] \cos (D \lambda) \\
+[a(\lambda, p, A)-b(\lambda, p, A)] \sin (D \lambda)) \mathrm{d} \lambda, \\
P_{2}(D, p, A):=\int_{\frac{1}{3}} \tilde{g}(\lambda) \lambda^{-1}([a(\lambda, p, A)+b(\lambda, p, A)] \sin (D \lambda) \\
\quad-[a(\lambda, p, A)-b(\lambda, p, A)] \cos (D \lambda)) \mathrm{d} \lambda .
\end{array}
$$

Lemma 16 There is a constant $C>0$ such that for all $D \in\left[-\frac{3 \pi}{4}, \frac{3 \pi}{4}\right], p \in$ $\left[-\frac{1}{4}, \frac{1}{4}\right]$ and $A>0$ at least one of the inequalities

$$
\left(\left|P_{1}(D, p, A)\right| \geq C\right) \quad \text { or } \quad\left(\left|P_{2}(D, p, A)\right| \geq C\right)
$$

is true.

Proof We define

$$
\begin{aligned}
P^{+}(D, p, A) & :=P_{1}(D, p, A)+P_{2}(D, p, A) \\
& =2 \int_{\frac{1}{3}}^{\frac{4}{3}} \tilde{g}(\lambda) \lambda^{-1}[b(\lambda, p, A) \cos (D \lambda)+a(\lambda, p, A) \sin (D \lambda)] \mathrm{d} \lambda
\end{aligned}
$$

and show that there exists a constant $C>0$ that either one of the statements in Eq. 38 or equivalently $\left(\left|P^{+}(D, p, A)\right| \geq C\right)$ is true. For the rest of the proof, the variables $p \in\left[-\frac{1}{4}, \frac{1}{4}\right]$ and $A>0$ will be arbitrary and fixed. For simplicity, we assume $D \in\left[0, \frac{3 \pi}{4}\right]$ since the proof for negative values of $D$ is similar. We consider different intervals for the variable $D$ and show that at least one of the equivalent 
propositions, see Eq. 38 or $\left(\left|P^{+}(D, p, A)\right| \geq C\right)$, is true. From the construction of the window function, we deduce $\tilde{g}(\lambda) \lambda^{-1}>0$ and from Lemma 15 we know $a(\lambda, p, A)+b(\lambda, p, A)>0$ for $\lambda \in\left[\frac{1}{3}, \frac{4}{3}\right]$. Thus, for $D=0$, it holds that

$$
P_{1}(0, p, A)=\int_{\frac{1}{3}}^{\frac{4}{3}} \tilde{g}(\lambda) \lambda^{-1}[a(\lambda, p, A)+b(\lambda, p, A)] \mathrm{d} \lambda>0 .
$$

For $D \in\left(0, \frac{3 \pi}{8}\right]$, we have $D \lambda \in\left(0, \frac{\pi}{2}\right)$ leading to $\sin (D \lambda)>0$ and $\cos (D \lambda)>0$ and from Lemma 15 we know $a(\lambda, p, A)>0$ and $b(\lambda, p, A)>0$ which gives $P^{+}(D, p, A)>0$.

$$
\begin{array}{r}
\text { For } D \in\left(\frac{3 \pi}{8}, \frac{3 \pi}{4}\right], \text { we split up } \int_{\frac{1}{3}}^{\frac{4}{3}}=\left(\int_{\frac{1}{3}}^{\frac{\pi}{2 D}}+\int_{\frac{\pi}{2 D}}^{\frac{\pi}{D}}+\int_{\frac{\pi}{D}}^{\frac{4}{3}}\right) \text { and write } \\
P_{2}(D, p, A):=P_{21}(D, p, A)+P_{22}(D, p, A)+P_{23}(D, p, A), \\
P^{+}(D, p, A):=P_{1}^{+}(D, p, A)+P_{2}^{+}(D, p, A)+P_{3}^{+}(D, p, A) .
\end{array}
$$

Since $\frac{4}{3} \leq \frac{\pi}{D}$ and supp $\widetilde{g}=\left[\frac{1}{3}, \frac{4}{3}\right]$, it follows that $P_{23}(D, p, A)=P_{3}^{+}(D, p, A)=0$.

We assume $a(\lambda, p, A)>b(\lambda, p, A)>0$ (the first case of Lemma 15). In the integral $P_{1}^{+}$we have $D \lambda \in\left(\frac{\pi}{8}, \frac{\pi}{2}\right)$, hence $\sin (D \lambda)>0$ and $\cos (D \lambda)>0$. This leads directly to $P_{1}^{+}(D, p, A)>0$. Since $D \lambda \in\left(\frac{\pi}{2}, \pi\right)$ implies $\cos (D \lambda)<0$ and $\sin (D \lambda)>0$ in the integral $P_{2}^{+}$we can estimate

$$
\begin{aligned}
P_{2}^{+}(D, p, A) & >\int_{\frac{\pi}{2 D}}^{\frac{\pi}{D}} \widetilde{g}(\lambda) \lambda^{-1} a(\lambda, p, A)(\cos (D \lambda)+\sin (D \lambda)) \mathrm{d} \lambda \\
& =\int_{\frac{\pi}{2 D}}^{\frac{\pi}{D}} \widehat{h}(\lambda, p, A)(\cos (D \lambda)+\sin (D \lambda)) \mathrm{d} \lambda
\end{aligned}
$$

with $\widehat{h}(\lambda, p, A):=\widetilde{g}(\lambda) \lambda^{-1} a(\lambda, p, A)$. This function is monotonically decreasing for $\lambda \in\left[\frac{2}{3}, \frac{4}{3}\right]$. Using the substitution $t=D \lambda-\frac{3 \pi}{4}$, we obtain

$$
\begin{aligned}
P_{2}^{+}(D, p, A) & >-\frac{\sqrt{2}}{D} \int_{-\frac{\pi}{4}}^{\frac{\pi}{4}} \widehat{h}\left(\frac{3 \pi+4 t}{4 D}, p, A\right) \sin t \mathrm{~d} t \\
& =\frac{\sqrt{2}}{D} \int_{0}^{\frac{\pi}{4}}\left[\widehat{h}\left(\frac{3 \pi-4 t}{4 D}, p, A\right)-\widehat{h}\left(\frac{3 \pi+4 t}{4 D}, p, A\right)\right] \sin t \mathrm{~d} t>0,
\end{aligned}
$$


where we used the monotonicity of the function $\widehat{h}$ to deduce the last inequality. Overall, for $D \in\left(\frac{3 \pi}{8}, \frac{3 \pi}{4}\right]$ and $a(\lambda, p, A)>b(\lambda, p, A)>0$ we showed $P_{1}^{+}(D, p, A)>$ $0, P_{2}^{+}(D, p, A)>0$ and $P_{3}^{+}(D, p, A)=0$, which leads to $P^{+}(D, p, A)>0$ in that case.

Let us assume $a(\lambda, p, A)+b(\lambda, p, A)>(1+\sqrt{2})|a(\lambda, p, A)-b(\lambda, p, A)|$ (the second case of Lemma 15). In $P_{21}$ we have $D \lambda \in\left(\frac{\pi}{8}, \frac{\pi}{2}\right)$, hence $(1+\sqrt{2}) \sin (D \lambda)>\cos (D \lambda)>0$, which allows for the estimate

$$
\begin{aligned}
P_{21}(D, p, A)> & \int_{\frac{1}{3}}^{\frac{\pi}{2 D}} \tilde{g}(\lambda) \lambda^{-1}|a(\lambda, p, A)-b(\lambda, p, A)| \\
& \times((1+\sqrt{2}) \sin (D \lambda)-\cos (D \lambda)) \mathrm{d} \lambda \\
> & 0 .
\end{aligned}
$$

To estimate the integral $P_{22}$ we can use exactly the same arguments as in Eqs. 39 and 40 but this time with the function $\widetilde{h}(\lambda, p, A):=\widetilde{g}(\lambda) \lambda^{-1}(a(\lambda, p, A)+b(\lambda, p, A))$ instead of $\widehat{h}$ which gives $P_{22}(D, p, A)>0$ and overall $P_{2}(D, p, A)>0$.

\section{Proof of the main results}

We start with the proof of Theorem 1 .

Recall that we denote the set of dyadic squares $Q \subseteq[-\pi, \pi)^{2}$ of the form Eq. 9 for $j \in \mathbb{N}_{0}$ by $\mathcal{Q}_{j}$ and smooth functions $\phi_{Q}$ with support on these dyadic squares with the property

$$
\sum_{Q \in \mathcal{Q}_{j}} \phi_{Q}(\mathbf{x})=1, \quad \mathbf{x} \in[-\pi, \pi)^{2},
$$

are defined in Eq. 18. Moreover, for $u \in \mathbb{N}$ let $f \in C^{u}\left(\mathbb{R}^{2}\right)$ and define $f_{Q}:=f \phi_{Q}$ for $Q \in \mathcal{Q}_{j}$. We can decompose

$$
f=\sum_{Q \in \mathcal{Q}_{j}} f_{Q}=\sum_{Q \in \mathcal{Q}_{j}^{0}} f_{Q}+\sum_{Q \in \mathcal{Q}_{j}^{1}} f_{Q},
$$

where $Q \in \mathcal{Q}_{j}^{1} \subseteq \mathcal{Q}_{j}$ if $\partial T \cap Q \neq \emptyset$. For the non-intersecting squares we define $\mathcal{Q}_{j}^{0}:=\mathcal{Q}_{j} \backslash \mathcal{Q}_{j}^{1}$.

According to Eq. 10, we denote by $f_{Q}^{2 \pi}$ the $2 \pi$-periodization of $f_{Q}$. From the finite support of $f_{Q}$, we deduce $f_{Q} \in L_{1}\left(\mathbb{R}^{2}\right)$ and from Eq. 15 we get

$$
c_{\mathbf{k}}\left(f_{Q}^{2 \pi}\right)=\mathcal{F}\left[f_{Q}\right](\mathbf{k}), \quad \mathbf{k} \in \mathbb{Z}^{2} .
$$

Moreover by Eq. 12, we have $\mathcal{F} f_{Q} \in C^{q}\left(\mathbb{R}^{2}\right)$ for all $q \in \mathbb{N}_{0}$. The smoothness assumption on the window function $\Psi_{j, \ell}^{(i)} \in \mathcal{W}_{2}^{2 q}$ implies $\mathcal{F}\left[f_{Q}\right] \Psi_{j, \ell}^{(i)} \in C_{0}^{q}\left(\mathbb{R}^{2}\right)$. 
Thus, the estimates Eq. 14 hold for this function and with Parseval's identity and the Poisson summation formula it follows:

$$
\begin{aligned}
\left\langle f_{Q}^{2 \pi}, \psi_{j, \ell, \mathbf{y}}^{(i)}\right\rangle_{2} & =\sum_{\mathbf{k} \in \mathbb{Z}^{2}} \mathcal{F}\left[f_{Q}\right](\mathbf{k}) \Psi_{j, \ell}^{(i)}(\mathbf{k}) \mathrm{e}^{2 \pi i \mathbf{k}^{\mathrm{T}} \tilde{\mathbf{y}}} \\
& =\sum_{\mathbf{n} \in \mathbb{Z}^{2}} \mathcal{F}^{-1}\left[\mathcal{F}\left[f_{Q}\right] \Psi_{j, \ell}^{(i)}\right](2 \pi(\widetilde{\mathbf{y}}+\mathbf{n}))=\sum_{\mathbf{n} \in \mathbb{Z}^{2}} S_{Q}(\mathbf{n}),
\end{aligned}
$$

where

$$
S_{Q}(\mathbf{n}):=\int_{\mathbb{R}^{2}} \mathcal{F}\left[f_{Q}\right](\boldsymbol{\xi}) \Psi_{j, \ell}^{(i)}(\boldsymbol{\xi}) \mathrm{e}^{2 \pi \mathrm{i} \xi^{\mathrm{T}}(\widetilde{\mathbf{y}}+\mathbf{n})} \mathrm{d} \boldsymbol{\xi} .
$$

For $Q \in \mathcal{Q}_{j}^{0}$, we choose $\mathbf{x}_{1} \in[-\pi, \pi]^{2}$ such that

$$
1 \leq\left|2 \pi \tilde{\mathbf{y}}-\mathbf{x}_{1}\right|_{\infty} \leq\left|2 \pi \tilde{\mathbf{y}}-\mathbf{x}_{1}\right|_{2} \leq \pi
$$

and define $\tilde{f}(\mathbf{x}):=f_{Q}\left(\mathbf{x}-\mathbf{x}_{1}\right)$. From Eq. 21, we see that $\mathcal{F}[\tilde{f}](\xi)=\mathrm{e}^{\mathrm{i} \mathbf{x}_{1}^{\mathrm{T}} \boldsymbol{\xi}} \mathcal{F}\left[f_{Q}\right](\boldsymbol{\xi})$ and since $\mathcal{F} \tilde{f} \Psi_{j, \ell}^{(i)} \in C_{0}^{q}\left(\mathbb{R}^{2}\right)$ we can use integration by parts repeatedly in both variables for every $\mathbf{r} \in \mathbb{N}_{0}^{2}$ with $|\mathbf{r}|_{1} \leq q$ to obtain

$$
\left(2 \pi \mathrm{i}(\widetilde{\mathbf{y}}+\mathbf{n})-\mathbf{x}_{1}\right)^{\mathbf{r}} S_{Q}(\mathbf{n})=\int_{\mathbb{R}^{2}} \partial^{\mathbf{r}}\left[\mathcal{F}[\widetilde{f}] \Psi_{j, \ell}^{(i)}\right](\boldsymbol{\xi}) \mathrm{e}^{\mathrm{i} \xi^{\mathrm{T}}\left(2 \pi(\widetilde{\mathbf{y}}+\mathbf{n})-\mathbf{x}_{1}\right)} \mathrm{d} \boldsymbol{\xi} .
$$

With the calculation

$$
\left(1+2^{j}\left|2 \pi(\widetilde{\mathbf{y}}+\mathbf{n})-\mathbf{x}_{1}\right|_{2}^{2}\right)^{q}=\sum_{s=0}^{q}\left(\begin{array}{l}
q \\
s
\end{array}\right) 2^{j s} \sum_{|\mathbf{r}|_{1}=s}\left(\begin{array}{l}
s \\
\mathbf{r}
\end{array}\right)\left(2 \pi(\widetilde{\mathbf{y}}+\mathbf{n})-\mathbf{x}_{1}\right)^{2 \mathbf{r}}
$$

and the representation Eq. 23 of the $q$-th order differential operator $L^{q}$ we have

$$
\left(1+2^{j}\left|2 \pi(\widetilde{\mathbf{y}}+\mathbf{n})-\mathbf{x}_{1}\right|_{2}^{2}\right)^{q} S_{Q}(\mathbf{n})=\int_{\mathbb{R}^{2}} L^{q}\left[\mathcal{F}[\tilde{f}] \Psi_{j, \ell}^{(i)}\right](\boldsymbol{\xi}) \mathrm{e}^{\mathrm{i} \xi^{\mathrm{T}}\left(2 \pi(\tilde{\mathbf{y}}+\mathbf{n})-\mathbf{x}_{1}\right)} \mathrm{d} \boldsymbol{\xi} .
$$

A consequence from Hölder's inequality for a set $A \subset \mathbb{R}^{2}$ with finite Lebesgue measure $|A|$, parameters $1 \leq p \leq s<\infty$ and a function $f \in L_{s}(A)$ is the estimate

$$
\|f\|_{A, p} \leq|A|^{(s-p) /(p s)}\|f\|_{A, s} .
$$

From Lemma 8, we conclude $L^{q}\left[\mathcal{F}[\tilde{f}] \Psi_{j, \ell}^{(i)}\right] \in L_{2}\left(\mathbb{R}^{2}\right)$ and with the estimate Eq. 44 for $p=1$ and $s=2$ together with the upper bound for the support size of $\Psi_{j, \ell}^{(\mathrm{i})}$ given by Eq. 7 we see that

$$
\left\|L^{q}\left[\mathcal{F}[\tilde{f}] \Psi_{j, \ell}^{(i)}\right]\right\|_{\mathbb{R}^{2}, 1} \leq 2^{3 j / 4}\left\|L^{q}\left[\mathcal{F}[\tilde{f}] \Psi_{j, \ell}^{(i)}\right]\right\|_{\mathbb{R}^{2}, 2} .
$$

Next, Eqs. 43 and 45 and Lemma 8 for $u=2$ imply

$$
\begin{aligned}
\left|\left\langle f_{Q}^{2 \pi}, \psi_{j, \ell, \mathbf{y}}^{(i)}\right\rangle_{2}\right| & \leq \sum_{\mathbf{n} \in \mathbb{Z}^{2}}\left\|L^{q}\left[\mathcal{F}[\tilde{f}] \Psi_{j, \ell}^{(i)}\right]\right\|_{\mathbb{R}^{2}, 1}\left(1+2^{j}\left|2 \pi(\widetilde{\mathbf{y}}+\mathbf{n})-\mathbf{x}_{1}\right|_{2}^{2}\right)^{-q} \\
& \leq C(q) 2^{-7 j / 4} \sum_{\mathbf{n} \in \mathbb{Z}^{2}}\left(1+2^{j}\left|2 \pi(\widetilde{\mathbf{y}}+\mathbf{n})-\mathbf{x}_{1}\right|_{2}^{2}\right)^{-q}
\end{aligned}
$$


We split up the infinite sum in the last line into

$$
\left(1+2^{j}\left|2 \pi \tilde{\mathbf{y}}-\mathbf{x}_{1}\right|_{2}^{2}\right)^{-q}+\sum_{\mathbf{n} \in \mathbb{Z}^{2} \backslash\{\mathbf{0}\}}\left(1+2^{j}\left|2 \pi(\widetilde{\mathbf{y}}+\mathbf{n})-\mathbf{x}_{1}\right|_{2}^{2}\right)^{-q},
$$

where due to Eq. 42 the summand corresponding to $\mathbf{n}=\mathbf{0}$ is bounded from above by $C(q) 2^{-j q}$. With the monotonicity of finite vector norms and the inverse triangle inequality, we get

$$
\begin{aligned}
\left|2 \pi(\widetilde{\mathbf{y}}+\mathbf{n})-\mathbf{x}_{1}\right|_{2} \geq\left|2 \pi(\widetilde{\mathbf{y}}+\mathbf{n})-\mathbf{x}_{1}\right|_{\infty} & \geq \pi\left(2|\mathbf{n}|_{\infty}-\left|2 \widetilde{\mathbf{y}}-\frac{\mathbf{x}_{1}}{\pi}\right|_{\infty}\right) \\
& \geq \pi\left(2|\mathbf{n}|_{\infty}-1\right)
\end{aligned}
$$

for $\mathbf{n} \neq \mathbf{0}$, because again with Eq. 42 we have $\left|2 \widetilde{\mathbf{y}}-\frac{\mathbf{x}_{1}}{\pi}\right|_{\infty} \leq 1$. Moreover the equation $\left|\left\{\mathbf{n} \in \mathbb{Z}^{2} ;|\mathbf{n}|_{\infty}=k, k \in \mathbb{N}\right\}\right|=8 k$ holds, leading to

$$
\begin{aligned}
\sum_{\mathbf{n} \in \mathbb{Z}^{2} \backslash\{\mathbf{0}\}}\left(1+2^{j}\left|2 \pi(\widetilde{\mathbf{y}}+\mathbf{n})-\mathbf{x}_{1}\right|_{2}^{2}\right)^{-q} & \leq C(q) 2^{-j q} \sum_{k=1}^{\infty} \sum_{|\mathbf{n}|_{\infty}=k}\left(2|\mathbf{n}|_{\infty}-1\right)^{-2 q} \\
& =C(q) 2^{-j q} \sum_{k=1}^{\infty} \frac{8 k}{(2 k-1)^{2 q}} \\
& \leq C_{2}(q) 2^{-j q}
\end{aligned}
$$

Using the splitting Eq. 47 and the corresponding upper bound Eq. 48 for the infinite sum in Eq. 46, we get:

$$
\left|\left\langle f_{Q}^{2 \pi}, \psi_{j, \ell, \mathbf{y}}^{(i)}\right\rangle_{2}\right| \leq C(q) 2^{-j(7 / 4+q)}
$$

in the case $Q \in \mathcal{Q}_{j}^{0}$.

For $Q \in \mathcal{Q}_{j}^{1}$, we use Eq. 21 to write

$$
S_{Q}(\mathbf{n}):=\int_{\mathbb{R}^{2}} \mathcal{F}\left[\mathcal{E}_{j}\right]\left(\mathbf{R}_{\gamma}^{\mathrm{T}} \boldsymbol{\xi}\right) \Psi_{j, \ell}^{(i)}(\boldsymbol{\xi}) \mathrm{e}^{\mathrm{i} \xi^{\mathrm{T}}\left(2 \pi(\widetilde{\mathbf{y}}+\mathbf{n})-\mathbf{x}_{0}\right)} \mathrm{d} \boldsymbol{\xi},
$$

where $\mathcal{E}_{j}$ is a standard edge fragment. With the same arguments as in the first case and again Lemma 8 , we can deduce:

$$
\begin{aligned}
\left|\left\langle f_{Q}^{2 \pi}, \psi_{j, \ell, \mathbf{y}}^{(i)}\right\rangle_{2}\right| & \leq \sum_{\mathbf{n} \in \mathbb{Z}^{2}}\left\|L^{q}\left[\mathcal{F}\left[\mathcal{E}_{j}\right]\left(\mathbf{R}_{\gamma}^{\mathrm{T}} \cdot\right) \Psi_{j, \ell}^{(i)}\right]\right\|_{\mathbb{R}^{2}, 1}\left(1+2^{j}\left|2 \pi(\widetilde{\mathbf{y}}+\mathbf{n})-\mathbf{x}_{0}\right|_{2}^{2}\right)^{-q} \\
& \leq C\left(1+2^{j / 2}\left|\sin \left(\theta_{j, \ell}^{(i)}-\gamma\right)\right|\right)^{-5 / 2} \\
& \times \sum_{\mathbf{n} \in \mathbb{Z}^{2}}\left(1+2^{j}\left|2 \pi(\widetilde{\mathbf{y}}+\mathbf{n})-\mathbf{x}_{0}\right|_{2}^{2}\right)^{-q}
\end{aligned}
$$


and we split up the infinite sum into

$$
\left(1+2^{j}\left|2 \pi \widetilde{\mathbf{y}}-\mathbf{x}_{0}\right|_{2}^{2}\right)^{-q}+\sum_{\mathbf{n} \in \mathbb{Z}^{2} \backslash\{\mathbf{0}\}}\left(1+2^{j}\left|2 \pi(\widetilde{\mathbf{y}}+\mathbf{n})-\mathbf{x}_{0}\right|_{2}^{2}\right)^{-q} .
$$

Using the same arguments, which led to Eq. 48, we see that the infinite sum in the last equation is bounded from above by $C(q) 2^{-j q}$ implying

$$
\left|\left\langle f_{Q}^{2 \pi}, \psi_{j, \ell, \mathbf{y}}^{(i)}\right\rangle_{2}\right| \leq C(q)\left(1+2^{j / 2}\left|\sin \left(\theta_{j, \ell}^{(i)}-\gamma\right)\right|\right)^{-5 / 2}\left(1+2^{j}\left|\mathbf{x}_{0}-2 \pi \widetilde{\mathbf{y}}\right|_{2}^{2}\right)^{-q}
$$

in the case $Q \in \mathcal{Q}_{j}^{1}$.

To finish the proof, we use the decomposition Eq. 41 and the fact that $\left|\mathcal{Q}_{j}^{0}\right| \leq C 2^{j}$ to get

$$
\begin{aligned}
\left|\left\langle f^{2 \pi}, \psi_{j, \ell, \mathbf{y}}^{(i)}\right\rangle_{2}\right| \leq & \sum_{Q \in \mathcal{Q}_{j}^{0}}\left|\left\langle f_{Q}^{2 \pi}, \psi_{j, \ell, \mathbf{y}}^{(i)}\right\rangle_{2}\right|+\sum_{Q \in \mathcal{Q}_{j}^{1}}\left|\left\langle f_{Q}^{2 \pi}, \psi_{j, \ell, \mathbf{y}}^{(i)}\right\rangle_{2}\right| \\
\leq & C_{3}(q) \sum_{Q \in \mathcal{Q}_{j}^{1}}\left(1+2^{j / 2}\left|\sin \left(\theta_{j, \ell}^{(i)}-\gamma\right)\right|\right)^{-5 / 2} \\
& \times\left(1+2^{j}\left|\mathbf{x}_{0}-2 \pi \widetilde{\mathbf{y}}\right|_{2}^{2}\right)^{-q} .
\end{aligned}
$$

We proceed with the proof of Theorem 2 .

For $\mathbf{y} \in \mathcal{P}\left(\mathbf{N}_{j, \ell}^{(i)}\right)$ let $\widehat{\mathcal{T}}_{\mathbf{y}}^{(i)}$ be the modified version of $\mathcal{T}^{(i)}$ as explained in the paragraph after Eq. 28. Since $\widehat{\mathcal{T}}_{\mathbf{y}}^{(i)} \in L_{1}\left(\mathbb{R}^{2}\right), i \in\{\mathrm{h}, \mathrm{v}\}$, we use Eq. 15 to get

$$
c_{\mathbf{k}}\left(\left(\widehat{\mathcal{T}}_{\mathbf{y}}^{(i)}\right)^{2 \pi}\right)=\mathcal{F} \widehat{\mathcal{T}}_{\mathbf{y}}^{(i)}(\mathbf{k}), \quad \mathbf{k} \in \mathbb{Z}^{2} .
$$

From the finite support of $\widehat{\mathcal{T}}_{\mathbf{y}}^{(i)}$, we deduce $\mathcal{F} \widehat{\mathcal{T}}_{\mathbf{y}}^{(i)} \in C^{2 q}\left(\mathbb{R}^{2}\right)$ for all $q \in \mathbb{N}_{0}$. The smoothness assumption on the window $\Psi_{j, \ell}^{(i)} \in \mathcal{W}_{2}^{2 q}$ implies $\mathcal{F}\left[\widehat{\mathcal{T}}_{\mathbf{y}}^{(i)}\right] \Psi_{j, \ell}^{(i)} \in$ $C_{0}^{2 q}\left(\mathbb{R}^{2}\right)$. Similar as in the proof of Theorem 1, this product fulfills Eq. 14 and with Parseval's identity and the Poisson summation formula it follows:

$$
\left\langle\left(\widehat{\mathcal{T}}_{\mathbf{y}}^{(i)}\right)^{2 \pi}, \psi_{j, \ell, \mathbf{y}}^{(i)}\right\rangle_{2}=\sum_{\mathbf{n} \in \mathbb{Z}^{2}} \mathcal{F}^{-1}\left[\mathcal{F}\left[\widehat{\mathcal{T}}_{\mathbf{y}}^{(i)}\right] \Psi_{j, \ell}^{(i)}\right](2 \pi(\widetilde{\mathbf{y}}+\mathbf{n}))=\sum_{\mathbf{n} \in \mathbb{Z}^{2}} S(\mathbf{n}),
$$

where

$$
S(\mathbf{n}):=\int_{\mathbb{R}^{2}} \mathcal{F}\left[\widehat{\mathcal{T}}_{\mathbf{y}}^{(i)}\right](\boldsymbol{\xi}) \Psi_{j, \ell}^{(i)}(\boldsymbol{\xi}) \mathrm{e}^{2 \pi \mathrm{i} \xi^{\mathrm{T}}(\widetilde{\mathbf{y}}+\mathbf{n})} \mathrm{d} \boldsymbol{\xi} .
$$

Using again the decomposition Eq. 41 for $\mathcal{T}$ we can repeat the arguments from the proof of Theorem 1 to see that

$$
\sum_{\mathbf{n} \in \mathbb{Z}^{2} \backslash\{\mathbf{0}\}}|S(\mathbf{n})| \leq C_{1}(q) 2^{-j q} .
$$

Assume that we can show

$$
|S(\mathbf{0})| \geq C_{2}(q)
$$


With the inverse triangle inequality, we can deduce

$$
\left|\left\langle\left(\widehat{\mathcal{T}}_{\mathbf{y}}^{(i)}\right)^{2 \pi}, \psi_{j, \ell, \mathbf{y}}^{(i)}\right\rangle_{2}\right| \geq|S(\mathbf{0})|-\sum_{\mathbf{n} \in \mathbb{Z}^{2} \backslash\{\mathbf{0}\}}|S(\mathbf{n})| \geq C_{3}(q)
$$

and again with the inverse triangle inequality and Lemma 10 we finally get

$$
\left|\left\langle\mathcal{T}^{2 \pi}, \psi_{j, \ell, \mathbf{y}}^{(i)}\right\rangle_{2}\right| \geq\left|\left\langle\left(\widehat{\mathcal{T}}_{\mathbf{y}}^{(i)}\right)^{2 \pi}, \psi_{j, \ell, \mathbf{y}}^{(i)}\right\rangle_{2}\right|-\left|\left\langle\mathcal{T}^{2 \pi}-\left(\widehat{\mathcal{T}}_{\mathbf{y}}^{(i)}\right)^{2 \pi}, \psi_{j, \ell, \mathbf{y}}^{(i)}\right\rangle_{2}\right| \geq C_{4}\left(A_{0}, q\right)
$$

Thus, it is left to show the existence of a constant $C_{2}(q)>0$ such that Eq. 50 is fulfilled.

From Eqs. 30 and 31, we recall the representation:

$$
S(\mathbf{0})=\frac{2^{j} \mathrm{i}}{(2 \pi)^{2}} \sum_{k=1}^{M} \int_{0}^{\infty} \int_{0}^{2 \pi} \Psi_{j, \ell}^{(i)}\left(2^{j} \rho, \theta\right) \mathrm{e}^{2 \pi \mathrm{i} 2^{j} \rho \boldsymbol{\Theta}^{\mathrm{T}}(\theta)} \tilde{\mathbf{y}} \mathcal{I}_{k}\left(2^{j} \rho, \theta\right) \mathrm{d} \theta \mathrm{d} \rho,
$$

and consider only the case $i=\mathrm{h}$ since the other case is similar. First, we use Lemma 11 and the inverse triangle inequality to see that $|S(\mathbf{0})|$ is bounded from below by

$$
\frac{2^{j}}{(2 \pi)^{2}}\left(\left|\sum_{k \in \mathcal{M}^{(\mathrm{v})}} \int_{0}^{\infty} \int_{0}^{2 \pi} \Psi_{j, \ell}^{(\mathrm{h})}\left(2^{j} \rho, \theta\right) \mathrm{e}^{2 \pi \mathrm{i} 2^{j} \rho \boldsymbol{\Theta}^{\mathrm{T}}(\theta) \widetilde{\mathbf{y}}_{\mathcal{I}}} \mathcal{I}_{k}\left(2^{j} \rho, \theta\right) \mathrm{d} \theta \mathrm{d} \rho\right|-\left|\mathcal{M}^{(\mathrm{h})}\right| C(n) 2^{-j n}\right),
$$

where the last term is negligible for large $j$ and $n \in \mathbb{N}$. Assume that there is $k^{*} \in$ $\mathcal{M}^{(\mathrm{v})}$ such that $\left[2 \pi y_{2}-\pi 2^{-j / 2}, 2 \pi y_{2}+2^{-j / 2}\right] \subseteq\left[a_{k^{*}}, b_{k^{*}}\right]$. In the following, we omit the index $k^{*}$ for simplicity and let $\varepsilon=2^{-j / 2}$. Lemma 12 and the inverse triangle inequality lead to

$$
\begin{aligned}
& \left|\sum_{k \in \mathcal{M}^{(\mathrm{v})}} \int_{0}^{\infty} \int_{0}^{2 \pi} \Psi_{j, \ell}^{(\mathrm{h})}\left(2^{j} \rho, \theta\right) \mathrm{e}^{2 \pi \mathrm{i} 2^{j} \rho \boldsymbol{\Theta}^{\mathrm{T}}(\theta)} \tilde{\mathbf{y}}_{\mathcal{I}}\left(2^{j} \rho, \theta\right) \mathrm{d} \theta \mathrm{d} \rho\right| \\
\geq & \left|\int_{0}^{\infty} \int_{0}^{2 \pi} \int_{2 \pi y_{2}-\varepsilon}^{2 \pi y_{2}+\varepsilon} \Psi_{j, \ell}^{(\mathrm{h})}\left(2^{j} \rho, \theta\right) \mathrm{e}^{\mathrm{i} 2^{j} \rho \boldsymbol{\Theta}^{\mathrm{T}}(\theta)\left(2 \pi \widetilde{\mathbf{y}}-(f(x), x)^{\mathrm{T}}\right)} \boldsymbol{\Theta}^{\mathrm{T}}(\theta) \boldsymbol{\beta}(x) \mathrm{d} x \mathrm{~d} \theta \mathrm{d} \rho\right| \\
& -C_{2} 2^{-j n} .
\end{aligned}
$$

From the previous observations, we conclude that, if we want to show $|S(\mathbf{0})| \geq C(q)$, it is enough to find a constant $C_{2}(q)>0$ such that

$$
\begin{aligned}
& \left|\int_{0}^{\infty} \int_{0}^{2 \pi} \int_{2 \pi y_{2}-\varepsilon}^{2 \pi y_{2}+\varepsilon} \Psi_{j, \ell}^{(\mathrm{h})}\left(2^{j} \rho, \theta\right) \mathrm{e}^{\mathrm{i} 2^{j} \rho \boldsymbol{\Theta}^{\mathrm{T}}(\theta)\left(2 \pi \tilde{\mathbf{y}}-(f(x), x)^{\mathrm{T}}\right)} \boldsymbol{\Theta}^{\mathrm{T}}(\theta) \boldsymbol{\beta}(x) \mathrm{d} x \mathrm{~d} \theta \mathrm{d} \rho\right| \\
& \geq C_{2}(q) 2^{-j} .
\end{aligned}
$$


We write the last integral as

$$
\begin{aligned}
I & :=\int_{0}^{\infty} \int_{0}^{2 \pi} \int_{2 \pi y_{2}-\varepsilon}^{2 \pi y_{2}+\varepsilon} \Psi_{j, \ell}^{(\mathrm{h})}\left(2^{j} \rho, \theta\right) \mathrm{e}^{\mathrm{i} 2^{j} \rho \boldsymbol{\Theta}^{\mathrm{T}}(\theta)\left(2 \pi \tilde{\mathbf{y}}-(f(x), x)^{\mathrm{T}}\right)} \boldsymbol{\Theta}^{\mathrm{T}}(\theta) \boldsymbol{\beta}(x) \mathrm{d} x \mathrm{~d} \theta \mathrm{d} \rho \\
& =\int_{0}^{\infty}\left(\int_{-\frac{\pi}{2}}^{\frac{\pi}{2}}+\int_{\frac{\pi}{2}}^{\frac{3 \pi}{2}} \int_{2 \pi y_{2}-\varepsilon}^{2 \pi y_{2}+\varepsilon} \Psi_{j, \ell}^{(\mathrm{h})}\left(2^{j} \rho, \theta\right) \mathrm{e}^{\mathrm{i} 2^{j} \rho \boldsymbol{\Theta}^{\mathrm{T}}(\theta)\left(2 \pi \tilde{\mathbf{y}}-(f(x), x)^{\mathrm{T}}\right)}\right. \\
& \times \boldsymbol{\Theta}^{\mathrm{T}}(\theta) \boldsymbol{\beta}(x) \mathrm{d} x \mathrm{~d} \theta \mathrm{d} \rho \\
& =: I_{1}+I_{2} .
\end{aligned}
$$

In the integral $I_{2}$, we substitute $\theta=\tau+\pi$ and use the symmetry properties of the univariate window functions $\widetilde{g}$ and $g$ to see

$$
\tilde{g}(\rho \cos (\tau+\pi))=\widetilde{g}(-\rho \cos \tau)=\widetilde{g}(\rho \cos \tau)
$$

and

$$
g\left(\rho \cos (\tau+\pi)\left(2^{j / 2} \tan (\tau+\pi)-\ell\right)\right)=g\left(\rho \cos \tau\left(2^{j / 2} \tan \tau-\ell\right)\right),
$$

which lead together with $\boldsymbol{\Theta}(\tau+\pi)=-\boldsymbol{\Theta}(\tau)$ to $I=2 \mathrm{i} \operatorname{Im}\left(I_{1}\right)=2 \mathrm{i} \operatorname{Im}\left(I_{2}\right)$ since

$$
\begin{aligned}
I_{2} & =\int_{0}^{\infty} \int_{-\frac{\pi}{2}}^{\frac{\pi}{2}} \int_{2 \pi}^{2 \pi y_{2}-\varepsilon} \Psi_{j, \ell}^{(\mathrm{h})}\left(2^{j} \rho, \tau+\pi\right) \mathrm{e}^{\mathrm{i} 2^{j} \rho \boldsymbol{\Theta}^{\mathrm{T}}(\tau+\pi)\left(2 \pi \tilde{\mathbf{y}}-(f(x), x)^{\mathrm{T}}\right)} \\
& \times \boldsymbol{\Theta}(\tau+\pi) \boldsymbol{\beta}(x) \mathrm{d} x \mathrm{~d} \tau \mathrm{d} \rho \\
& =-\int_{0}^{\infty} \int_{-\frac{\pi}{2}}^{\frac{\pi}{2}} \int_{2 \pi y_{2}-\varepsilon}^{2 \pi y_{2}+\varepsilon} \Psi_{j, \ell}^{(\mathrm{h})}\left(2^{j} \rho, \theta\right) \mathrm{e}^{-\mathrm{i} 2^{j} \rho \boldsymbol{\Theta}^{\mathrm{T}}(\theta)\left(2 \pi \tilde{\mathbf{y}}-(f(x), x)^{\mathrm{T}}\right)} \\
& \times \boldsymbol{\Theta}(\theta) \boldsymbol{\beta}(x) \mathrm{d} x \mathrm{~d} \theta \mathrm{d} \rho=-\overline{I_{1}} .
\end{aligned}
$$

Let us first assume $A=\frac{1}{2} f^{\prime \prime}\left(2 \pi y_{2}\right)>0$. The case $A<0$ is similar and will be omitted and the case $A=0$ will be discussed separately in the end of the proof. With Lemma 10 we can replace the function $f(x)$ locally for $\left|x-2 \pi y_{2}\right|<2^{-j / 2}$ by

$$
T_{\mathbf{y}}(x)=f\left(2 \pi y_{2}\right)+m\left(x-2 \pi y_{2}\right)+A\left(x-2 \pi y_{2}\right)^{2} .
$$

For every $\mathbf{x}_{0}=\left(f\left(x_{0}\right), x_{0}\right)^{\mathrm{T}} \in \partial T$ with $\left|\mathbf{x}_{0}-2 \pi \tilde{\mathbf{y}}\right|_{2} \leq C 2^{-j / 2}$ we can write

$$
T_{\mathbf{y}}(x)=C+B\left(x-x_{0}\right)+A\left(x-x_{0}\right)^{2},
$$

where $B:=m+2\left(x_{0}-2 \pi y_{2}\right), C:=f\left(2 \pi y_{2}\right)+m\left(x_{0}-2 \pi y_{2}\right)+\left(x_{0}-2 \pi y_{2}\right)^{2}$ and $m=$ $f^{\prime}\left(2 \pi y_{2}\right) \in[-1,1]$. We choose $\mathbf{x}_{0} \in \partial T$ such that there is $\ell \in\left\{-2^{j / 2}, \ldots, 2^{j / 2}\right\}$ with $\left|2^{j / 2} B+\ell\right| \leq \frac{1}{4}$ and $\tilde{y}_{1}=2^{-j}\left(z_{1}-\frac{1}{2}\right), z_{1}=-2^{j-1}, \ldots, 2^{j-1}-1$ such that $\left|2^{j}\left(2 \pi \tilde{y}_{1}-C\right)\right| \leq \frac{3 \pi}{4}$. 
We follow the ideas of [17, Section 3.2] and change the variable to $v=x-x_{0}$. Thus, we can rewrite $I_{1}$ as

$$
\begin{aligned}
I_{1} & =\int_{0}^{\infty} \int_{-\frac{\pi}{2}}^{\frac{\pi}{2}} \int_{-2 \varepsilon}^{2 \varepsilon} \Psi_{j, \ell}^{(\mathrm{h})}\left(2^{j} \rho, \theta\right) \mathrm{e}^{-\mathrm{i} 2^{j} \rho \boldsymbol{\Theta}^{\mathrm{T}}(\theta)\left(A v^{2}+B v+C-2 \pi \tilde{y}_{1}, v\right)^{\mathrm{T}}} \varphi(v) \mathrm{d} v \mathrm{~d} \theta \mathrm{d} \rho \\
& =\int_{0}^{\infty} \int_{-\frac{\pi}{2}}^{\frac{\pi}{2}} \Psi_{j, \ell}^{(\mathrm{h})}\left(2^{j} \rho, \theta\right) \mathrm{e}^{\mathrm{i} 2^{j} \rho \cos \theta 2 \pi \widetilde{y}_{1}} \int_{-2 \varepsilon}^{2 \varepsilon} \mathrm{e}^{\mathrm{i} \Lambda R(v)} \varphi(v) \mathrm{d} v \mathrm{~d} \theta \mathrm{d} \rho,
\end{aligned}
$$

where $\Lambda:=2^{j} \rho, \varphi(v):=\left(-1, f^{\prime}\left(v+x_{0}\right)\right) \boldsymbol{\Theta}(\theta) \beta\left(v+x_{0}\right)$ and

$$
\begin{aligned}
R(v) & :=-\boldsymbol{\Theta}^{\mathrm{T}}(\theta)\left(A v^{2}+B v+C, v\right)^{\mathrm{T}} \\
& =-\cos \theta\left(A\left(v+\frac{B+\tan \theta}{2 A}\right)^{2}+C-\frac{(B+\tan \theta)^{2}}{4 A}\right) .
\end{aligned}
$$

The equation $R^{\prime}(v)=0$ gives $v_{\theta}=-\frac{B+\tan \theta}{2 A}$. Let $\phi(v)=R(v)-R\left(v_{\theta}\right)$. Then $\phi\left(v_{\theta}\right)=\phi^{\prime}\left(v_{\theta}\right)=0$ and $\phi^{\prime \prime}\left(v_{\theta}\right)=R^{\prime \prime}\left(v_{\theta}\right)=-2 A \cos \theta \neq 0$. Hence, we can write $I_{1}$ as

$$
I_{1}=\int_{0}^{\infty} \int_{-\frac{\pi}{2}}^{\frac{\pi}{2}} \Psi_{j, \ell}^{(\mathrm{h})}\left(2^{j} \rho, \theta\right) \mathrm{e}^{\mathrm{i} 2^{j} \rho \cos \theta 2 \pi \tilde{y}_{1}} \mathrm{e}^{\mathrm{i} \Lambda R\left(v_{\theta}\right)} \int_{-2 \varepsilon}^{2 \varepsilon} \mathrm{e}^{\mathrm{i} \Lambda \phi(v)} \varphi(v) \mathrm{d} v \mathrm{~d} \theta \mathrm{d} \rho .
$$

We apply Lemma 13 for $t_{0}=v_{\theta}$, which leads to

$$
\begin{aligned}
\int_{-2 \varepsilon}^{2 \varepsilon} \mathrm{e}^{\mathrm{i} \Lambda \phi(v)} \varphi(v) \mathrm{d} v & =\left(\frac{2 \pi \mathrm{i}}{\left|\phi^{\prime \prime}\left(v_{\theta}\right)\right|}\right)^{1 / 2} \varphi\left(v_{\theta}\right) \Lambda^{-\frac{1}{2}}+O\left(\Lambda^{-1}\right) \\
& =C \sqrt{\pi \mathrm{i}}\left(2^{j} \rho|A \cos \theta|\right)^{-\frac{1}{2}} \varphi\left(v_{\theta}\right)+O\left(\left(2^{j} \rho\right)^{-1}\right) .
\end{aligned}
$$

From Lemma 1 , we have $\rho \in\left[\frac{1}{2}, 2\right]$ so that the notation $O\left(\left(2^{j} \rho\right)^{-1}\right)$ can be identified with a function $r(j)$ such that $|r(j)| \leq C_{2} 2^{-j}$ as $j \geq j_{0}$. As explained in [17, p. 115] the constant $C_{2}>0$ is independent of $\theta, \rho, j, \ell, \widetilde{\mathbf{y}}$. With Eq. 53 we can write the integral in Eq. 52 as $I_{1}=I_{11}+I_{12}$, where

$$
\begin{aligned}
I_{11}= & C 2^{-j / 2} \sqrt{\pi \mathrm{i}} \int_{0}^{\infty} \int_{-\frac{\pi}{2}}^{\frac{\pi}{2}} \Psi_{j, \ell}^{(\mathrm{h})}\left(2^{j} \rho, \theta\right) \mathrm{e}^{\mathrm{i} 2^{j} \rho \cos \theta 2 \pi \tilde{y}_{1}} \mathrm{e}^{\mathrm{i} \Lambda R\left(v_{\theta}\right)} \\
& \times(\rho|A \cos \theta|)^{-\frac{1}{2}} \varphi\left(v_{\theta}\right) \mathrm{d} \theta \mathrm{d} \rho, \\
I_{12}= & C_{2} 2^{-j} \int_{0}^{\infty} \int_{-\frac{\pi}{2}}^{\frac{\pi}{2}} \Psi_{j, \ell}^{(\mathrm{h})}\left(2^{j} \rho, \theta\right) \mathrm{e}^{\mathrm{i} 2^{j} \rho \cos \theta 2 \pi \tilde{y}_{1}} \mathrm{e}^{\mathrm{i} \Lambda R\left(v_{\theta}\right)} \mathrm{d} \theta \mathrm{d} \rho .
\end{aligned}
$$


In the integrals $I_{11}$ and $I_{12}$ we substitute $t=2^{j / 2} \tan \theta-\ell$ or equivalently $\theta:=\theta_{t}=$ $\theta_{j, \ell+t}^{(\mathrm{h})}$ leading to $\mathrm{d} \theta=2^{-j / 2} \cos ^{2} \theta_{t} \mathrm{~d} t$. We remind that by Lemma 1

$$
\operatorname{supp} \Psi_{j, \ell}^{(\mathrm{h})}\left(2^{j} \rho, \theta_{t}\right) \subset\left\{(\rho, \theta) \in \mathbb{R} \times\left[-\frac{\pi}{2}, \frac{\pi}{2}\right]: \frac{1}{3}<|\rho|<2, \theta_{j, \ell-2}^{(\mathrm{h})}<\theta_{t}<\theta_{j, \ell+2}^{(\mathrm{h})}\right\}
$$

implying that $I_{11}=I_{12}=0$ for $|t|>2$. With the last change of variable we have

$$
\begin{aligned}
I_{11}= & C 2^{-j} \sqrt{\pi \mathrm{i}} \int_{\frac{1}{3}}^{2} \int_{-2}^{2} \widetilde{g}\left(\rho \cos \theta_{t}\right) g\left(t \rho \cos \theta_{t}\right) \\
& \mathrm{e}^{-\mathrm{i} \rho \cos \theta_{t}\left(2^{j} C-\frac{1}{4 A}\left(2^{j / 2} B+\ell+t\right)^{2}-2^{j} 2 \pi \tilde{y}_{1}\right)} \\
& \times\left(\rho\left|A \cos \theta_{t}\right|\right)^{-\frac{1}{2}} \varphi\left(v_{\theta_{t}}\right) \cos ^{2} \theta_{t} \mathrm{~d} t \mathrm{~d} \rho, \\
I_{12}= & 2^{-3 j / 2} \int_{\frac{1}{3}}^{2} \int_{-2}^{2} \Psi_{j, \ell}^{(\mathrm{h})}\left(2^{j} \rho, \theta\right) \mathrm{e}^{-\mathrm{i} \rho \cos \theta_{t}\left(2^{j} C-\frac{1}{4 A}\left(2^{j / 2} B+\ell+t\right)^{2}-2^{j} 2 \pi \tilde{y}_{1}\right)} \cos ^{2} \theta_{t} \mathrm{~d} t \mathrm{~d} \rho .
\end{aligned}
$$

It is straightforward to see that $I_{12}$ is negligible since

$$
\left|I_{12}\right| \leq C 2^{-3 j / 2},
$$

where $C$ is independent of $j, \ell$, and $\mathbf{y}$. We use the notation

$$
p:=2^{j / 2} B+\ell, \quad D:=2^{j}\left(2 \pi \tilde{y}_{1}-C\right)
$$

and from the choice of $\mathbf{x}_{0} \in \partial T$ we have $|p| \leq \frac{1}{4}$ and $|D| \leq \frac{3 \pi}{4}$. We show that for this choice inequality Eq. 50 is fulfilled.

In the following, we adapt some of the ideas from [17]. Since $\left|2^{-j} t\right| \leq 2^{-j+1}$ for $|t| \leq 2$ we have $\cos \theta_{t}=\mu_{j, \ell}+O\left(2^{-j / 2}\right)$ and $\sin \theta_{t}=\left(2^{-j / 2} \ell\right) \mu_{j, \ell}+O\left(2^{-j / 2}\right)$, where $\mu_{j, \ell}:=\left(1+\left(2^{-j / 2} \ell\right)^{2}\right)^{-1 / 2}$ fulfilling $2^{-1 / 2} \leq\left|\mu_{j, \ell}\right| \leq 1$. There exists sufficiently small $q_{j, \ell}$ such that $\left|\beta\left(q_{j, \ell}\right)-\beta\left(v_{\theta_{t}}\right)\right|=O\left(2^{-j / 2}\right)$ and $\beta\left(q_{j, \ell}\right) \neq 0$ and similarly we can approximate $\left|f^{\prime}\left(\tilde{q}_{j, \ell}\right)-f^{\prime}\left(v_{\theta_{t}}\right)\right|=O\left(2^{-j / 2}\right)$ and $f^{\prime}\left(\tilde{q}_{j, \ell}\right) \neq 0$. To get the lower bound for $I_{11}$, after ignoring the higher order decay term we can replace $\beta\left(v_{\theta_{t}}\right)$ by a constant $\beta\left(q_{j, \ell}\right), f^{\prime}\left(v_{\theta_{t}}\right)$ by a constant $f^{\prime}\left(\tilde{q}_{j, \ell}\right)$, and $\cos \theta_{t}$ by the constant $\mu_{j, \ell}$. Hence, using the notation $\delta_{j, \ell}:=\beta\left(q_{j, \ell}\right)\left(-\mu_{j, \ell}+f^{\prime}\left(\tilde{q}_{j, \ell}\right)\left(2^{-j / 2} \ell\right) \mu_{j, \ell}\right)$ and the substitution $\lambda=\rho \mu_{j, \ell}$, we can express $I_{11}$ as

$$
\begin{aligned}
I_{11}(D, p) & =C 2^{-j} \mu_{j, \ell}^{3 / 2} \sqrt{\frac{\pi \mathrm{i}}{A}} \int_{\frac{1}{3}}^{2} \int_{-\infty}^{\infty} \tilde{g}\left(\rho \mu_{j, \ell}\right) \mathrm{e}^{\mathrm{i} \rho \mu_{j, \ell}\left(D+\frac{1}{4 A}(p+t)^{2}\right)} \\
& \times g\left(t \rho \mu_{j, \ell}\right) \rho^{-1 / 2} \mathrm{~d} t \mathrm{~d} \rho \\
& =C_{2} 2^{-j} \sqrt{\frac{\mathrm{i}}{A}} \int_{\frac{1}{3}}^{\frac{4}{3}} \tilde{g}(\lambda) \mathrm{e}^{\mathrm{i} D \lambda} \lambda^{-1 / 2} H(\lambda, p, A) \mathrm{d} \lambda
\end{aligned}
$$


with

$$
H(\lambda, p, A):=\int_{-\infty}^{\infty} g(t \lambda) \mathrm{e}^{\mathrm{i} \lambda \frac{1}{4 A}(p+t)^{2}} \mathrm{~d} t=\int_{-\infty}^{\infty} g((u-p) \lambda) \mathrm{e}^{\mathrm{i} \lambda \frac{u^{2}}{4 A}} \mathrm{~d} u
$$

We want to emphasize the dependency of the integral $I_{11}$ on the the parameters $p$ and $D$ defined in Eq. 55. A direct computation with the change of variable $v=\lambda \frac{u^{2}}{4 A}$ shows that

$$
\begin{aligned}
H(\lambda, p, A) & =\sqrt{\frac{A}{\lambda}} \int_{0}^{\infty}[g(2 \sqrt{A \lambda v}+p \lambda)+g(2 \sqrt{A \lambda v}-p \lambda)] \frac{\mathrm{e}^{\mathrm{i} v}}{\sqrt{v}} \mathrm{~d} v \\
& =\sqrt{\frac{A}{\lambda}}(a(\lambda, p, A)+\mathrm{i} b(\lambda, p, A)),
\end{aligned}
$$

where $a(\lambda, p, A)$ and $b(\lambda, p, A)$ are defined in Eqs. 35 and 36 . With the representation of $H(\lambda, p, A)$ and the positive solution $\sqrt{\mathrm{i}}=\frac{1+\mathrm{i}}{\sqrt{2}}$ we can write the integral Eq. 57 as $I_{11}(D, p)=\operatorname{Re}\left(I_{11}(D, p)\right)+\mathrm{i} \operatorname{Im}\left(I_{11}(D, p)\right)$ with

$$
\begin{gathered}
\operatorname{Im}\left(I_{11}(D, p)\right)=C 2^{-j} \int_{\frac{1}{3}}^{\frac{4}{3}} \widetilde{g}(\lambda) \lambda^{-1}([a(\lambda, p, A)+b(\lambda, p, A)] \cos (D \lambda) \\
+[a(\lambda, p, A)-b(\lambda, p, A)] \sin (D \lambda)) \mathrm{d} \lambda .
\end{gathered}
$$

Using the connection $I_{2}=-\overline{I_{1}}$, we can start at Eq. 51, use again Eq. 13 and repeat all the previous steps for $I_{2}$ instead of $I_{1}$, to get $I_{2}=I_{21}+I_{22}$ with $\left|I_{22}\right| \leq C 2^{-3 j / 2}$ and $I_{21}(D, p)=\operatorname{Re}\left(I_{21}(D, p)\right)+\mathrm{i} \operatorname{Im}\left(I_{21}(D, p)\right)$ with

$$
\begin{gathered}
\operatorname{Im}\left(I_{21}(D, p)\right)=C 2^{-j} \int_{\frac{1}{3}}^{\frac{4}{3}} \widetilde{g}(\lambda) \lambda^{-1}([a(\lambda, p, A)+b(\lambda, p, A)] \sin (D \lambda) \\
-[a(\lambda, p, A)-b(\lambda, p, A)] \cos (D \lambda)) \mathrm{d} \lambda .
\end{gathered}
$$

As a consequence of the relation $I=2 \mathrm{i} \operatorname{Im}\left(I_{1}\right)=2 \mathrm{i} \operatorname{Im}\left(I_{2}\right)$ we see that $I=$ $2 \mathrm{i} \operatorname{Im}\left(I_{11}+I_{12}\right)=2 \mathrm{i} \operatorname{Im}\left(I_{21}+I_{22}\right)$. By the inverse triangle inequality, Eq. 54 and its analog for $I_{22}$ we can use Lemma 16 in order to finish the proof of the Theorem 2 for $A>0$.

In the case $A=0$ we see that Eq. 51 simplifies to

$$
I_{1}=\int_{-\frac{\pi}{2}}^{\frac{\pi}{2}} \int_{-2 \varepsilon}^{2 \varepsilon} \Psi_{j, \ell}^{(\mathrm{h})}\left(2^{j} \rho, \theta\right) \mathrm{e}^{-\mathrm{i} \rho \cos \theta\left(2^{j / 2} v\left(2^{j / 2} B+2^{j / 2} \tan \theta\right)+2^{j}\left(C-2 \pi \tilde{y}_{1}\right)\right)} \varphi(v) \mathrm{d} v \mathrm{~d} \theta \mathrm{d} \rho .
$$

Note that Lemma 13 can not be applied in this case. Instead we use the substitutions $u=2^{j / 2} v$ and similar to the previous case $t=2^{j / 2} \tan \theta-\ell$ and $\lambda=\rho \mu_{j, \ell}$ together 
with the simplifications and notations from the last pages to arrive at the analogous integral to Eq. 57, which in this case is given by

$$
\begin{aligned}
I_{1}(D, p) & =C 2^{-j} \int_{0}^{\infty} \int_{-2}^{2} \int_{-\infty}^{\infty} \tilde{g}\left(\rho \cos \theta_{t}\right) g\left(t \rho \cos \theta_{t}\right) \mathrm{e}^{-\mathrm{i} \rho \cos \theta_{t}((p+t) u-D)} \mathrm{d} u \mathrm{~d} t \mathrm{~d} \rho \\
& =C 2^{-j} \int_{0}^{\infty} \int_{-2}^{2} \int_{-\infty}^{\infty} \widetilde{g}(\lambda) g(t \lambda) \mathrm{e}^{-\mathrm{i} \lambda((p+t) u-D)} \mathrm{d} u \mathrm{~d} t \mathrm{~d} \lambda .
\end{aligned}
$$

Some direct calculations after the change of variable $y=t \lambda$ show that

$$
\begin{aligned}
I_{1}(D, p) & =C 2^{-j} \int_{0}^{\infty} \tilde{g}(\lambda) \lambda^{-1} \mathrm{e}^{\mathrm{i} D \lambda} \int_{-\infty}^{\infty}\left(\int_{-\infty}^{\infty} g(y) \mathrm{e}^{-\mathrm{i} y u} \mathrm{~d} y\right) \mathrm{e}^{-\mathrm{i} p \lambda u} \mathrm{~d} u \mathrm{~d} \lambda \\
& =C 2^{-j} \int_{0}^{\infty} \tilde{g}(\lambda) \lambda^{-1} \mathrm{e}^{\mathrm{i} D \lambda}\left(\int_{-\infty}^{\infty} \mathcal{F} g(u) \mathrm{e}^{-\mathrm{i} p \lambda u} \mathrm{~d} u\right) \mathrm{d} \lambda \\
& =C 2^{-j} \int_{0}^{\infty} \tilde{g}(\lambda) \lambda^{-1} \mathrm{e}^{\mathrm{i} D \lambda} g(-p \lambda) \mathrm{d} \lambda
\end{aligned}
$$

and since $g(-p \lambda)=1$ for $\lambda \in\left[\frac{1}{3}, \frac{4}{3}\right]$ and $p \in\left[-\frac{1}{4}, \frac{1}{4}\right]$ this implies

$$
\left|\operatorname{Im}\left(I_{1}(D, p)\right)\right|=C 2^{-j} \int_{0}^{\infty} \tilde{g}(\lambda) \lambda^{-1} \sin (D \lambda) \mathrm{d} \lambda>0
$$

for $0<|D| \leq \frac{3 \pi}{4}$. For the case $D=0$, we slightly modify the function $\widetilde{g}$ to make it odd. Then with a similar argument as before we see that $I=2 \operatorname{Re}\left(I_{1}(0, p)\right)>0$.

\section{Generalizations and possible extensions}

In this paper, we showed that trigonometric polynomial shearlets based on the construction of multivariate periodic de la Vallée Poussin-type wavelets are able to detect step discontinuities along boundary curves of characteristic functions.

Since the constructions and results in [3] are given in $d$ dimensions, there is a natural extension of the trigonometric polynomial shearlets to higher dimensions. If for example the dimension is $d=3$, the multivariate window functions become $\Psi^{(1)}(\mathbf{x}):=\widetilde{g}\left(x_{1}\right) g\left(x_{2}\right) g\left(x_{3}\right), \quad \Psi^{(2)}(\mathbf{x}):=g\left(x_{1}\right) \widetilde{g}\left(x_{2}\right) g\left(x_{3}\right), \quad \Psi^{(3)}(\mathbf{x}):=g\left(x_{1}\right)$ $g\left(x_{2}\right) \widetilde{g}\left(x_{3}\right)$. 
For even $j \in \mathbb{N}_{0}$ and $\ell=\left(\ell_{1}, \ell_{2}\right)^{\mathrm{T}} \in \mathbb{Z}^{2}$ with $\left|\ell_{1}\right| \leq 2^{j / 2}$ and $\left|\ell_{2}\right| \leq 2^{j / 2}$ the matrices analog to Eq. 5 are given by

$$
\mathbf{N}_{j, \ell}^{(1)}:=\left(\begin{array}{ccc}
2^{j} & \ell_{1} 2^{j / 2} & \ell_{2} 2^{j / 2} \\
0 & 2^{j / 2} & 0 \\
0 & 0 & 2^{j / 2}
\end{array}\right), \quad \quad \mathbf{N}_{j, \ell}^{(2)}:=\left(\begin{array}{ccc}
2^{j / 2} & 0 & 0 \\
\ell_{1} 2^{j / 2} & 2^{j} & \ell_{2} 2^{j / 2} \\
0 & 0 & 2^{j / 2}
\end{array}\right)
$$

and

$$
\mathbf{N}_{j, \ell}^{(3)}:=\left(\begin{array}{ccc}
2^{j / 2} & 0 & 0 \\
0 & 2^{j / 2} & 0 \\
\ell_{1} 2^{j / 2} & \ell_{2} 2^{j / 2} & 2^{j}
\end{array}\right)
$$

We define the three-dimensional trigonometric polynomial shearlets by

$$
\psi_{j, \ell, \mathbf{y}}^{(i)}(\mathbf{x}):=\sum_{\mathbf{k} \in \mathbb{Z}^{3}} \Psi_{j, \ell}^{(i)}(\mathbf{k}) \mathrm{e}^{i \mathbf{k}^{\mathrm{T}}(\mathbf{x}-2 \pi \widetilde{\mathbf{y}})}, \quad i \in\{1,2,3\} .
$$

As in the two-dimensional case, this construction is similar to the classical shearlets and its higher-dimensional generalizations.

The authors in [15] proved in detail that continuous shearlet systems in three dimensions are able to detect boundary curves of piecewise smooth surfaces. As remarked in [17], an analogous result holds for discrete shearlets in dimension 3. We are convinced that it should be possible to derive a similar result for trigonometric polynomial shearlets, but a detailed proof is not in the focus of this paper.

Another interesting open question is the behavior of the shearlet coefficients near corner points. If $\boldsymbol{\gamma}:[0,2 \pi) \rightarrow \partial T$ is a parametrization of the boundary $\partial T$, we call $\mathbf{x}_{0}=\boldsymbol{\gamma}\left(t_{0}\right) \in \partial T$ a corner point, if $\boldsymbol{\gamma}^{\prime}\left(t_{0}^{+}\right) \neq \pm \boldsymbol{\gamma}^{\prime}\left(t_{0}^{-}\right)$. For continuous shearlets, this question was answered in [14] and in a more general setting in the context of parabolic molecules in [10]. As far as we know, there is no result for corner points in the discrete setting until now. It would be very interesting to investigate in which way the techniques of the continuous setting can be combined with the ideas of this paper to prove similar results for discrete shearlets. We will leave this question as a topic for future research.

In many applications, such as image processing, the functions to be analyzed are piecewise smooth and not characteristic functions of sets as discussed in this paper. In $[10,16]$, it was shown that the continuous shearlet coefficients of functions of the form $B(\mathbf{x})=f(\mathbf{x}) \chi_{T}(\mathbf{x})$ with $f \in C^{\infty}\left(\mathbb{R}^{2}\right)$ exhibit the same decay rate as Eq. 1 if $\mathbf{p} \notin \partial T$ or if $s=s_{0}$ does not correspond to the normal direction of $\partial T$ at $\mathbf{p}$. If $\mathbf{p} \in \partial T$ and $s=s_{0}$ corresponds to the normal direction of $\partial T$ at $\mathbf{p}$, then

$$
0<\lim _{a \rightarrow 0^{+}} a^{-(n / 2+3 / 4)} \mathcal{S} \mathcal{H}_{\psi} B\left(a, s_{0}, \mathbf{p}\right)<\infty,
$$

where $n$ denotes the number of vanishing derivatives of $f$ at $\mathbf{p}$. As in the case of corner points, there is no analogous result for discrete shearlet systems yet. To give a proof for the case of piecewise smooth functions is again beyond the scope of this paper and will be addressed in a forthcoming article.

Acknowledgments We would like to thank the referees for their valuable comments and remarks. 
Funding The authors were supported by H2020-MSCA-RISE-2014 Project number 645672 (AMMODIT: Approximation Methods for Molecular Modelling and Diagnosis Tools). Open Access funding enabled and organized by Projekt DEAL.

Open Access This article is licensed under a Creative Commons Attribution 4.0 International License, which permits use, sharing, adaptation, distribution and reproduction in any medium or format, as long as you give appropriate credit to the original author(s) and the source, provide a link to the Creative Commons licence, and indicate if changes were made. The images or other third party material in this article are included in the article's Creative Commons licence, unless indicated otherwise in a credit line to the material. If material is not included in the article's Creative Commons licence and your intended use is not permitted by statutory regulation or exceeds the permitted use, you will need to obtain permission directly from the copyright holder. To view a copy of this licence, visit http://creativecommonshorg/licenses/by/4. $0 /$.

\section{References}

1. Batenkov, D., Yomdin, Y.: Algebraic Fourier reconstruction of piecewise smooth functions. Math. Comput. 81, 277-318 (2011)

2. Bergmann, R.: The fast Fourier transform and fast wavelet transform for patterns on the torus. Appl. Comput. Harmon. Anal. 35, 39-51 (2013)

3. Bergmann, R., Prestin, J.: Multivariate periodic wavelets of de la vallée Poussin type. J. Fourier Anal. Appl. 21, 342-369 (2015)

4. Candès, E.J., Donoho, D.L.: New tight frames of curvelets and optimal representations of objects with piecewise $C^{2}$ singularities. Commun. Pure Appl. Math. 57, 219-266 (2004)

5. Dahlke, S., Steidl, G., Teschke, G.: The continuous shearlet transform in arbitrary space dimensions. J. Fourier Anal. Appl. 16, 340-364 (2010)

6. Eckhoff, K.S.: On a high order numerical method for functions with singularities. Math. Comput. 67, 1063-1087 (1998)

7. Gelb, A., Tadmor, E.: Detection of edges in spectral data. Appl. Comput. Harmon. Anal. 7, 101-135 (1999)

8. Goh, S.S., Lee, S.L., Teo, K.M.: Multidimensional periodic multiwavelets. J. Approx. Theory 98, 72-103 (1999)

9. Grohs, P.: Continuous shearlet frames and resolution of the wavefront set. Monatsh. Math. 164, 393426 (2011)

10. Grohs, P., Kereta, Z.: Analysis of edge and corner points using parabolic dictionaries. Appl. Comput. Harmon. Anal. 48, 655-681 (2020)

11. Guo, K., Kutyniok, G., Labate, D.: Sparse Multidimensional Representations Using Anisotropic Dilation and Shear Operators. In: Chen, G., Lai, M. (eds.) Wavelets and Splines, pp. 189-201. Nashboro Press (2006)

12. Guo, K., Labate, D.: Optimally sparse multidimensional representation using shearlets. SIAM J. Math. Anal. 39, 298-318 (2007)

13. Guo, K., Labate, D., Lim, W.Q.: Edge analysis and identification using the continuous shearlet transform. Appl. Comput. Harmon. Anal. 27, 24-46 (2009)

14. Guo, K., Labate, D.: Characterization and analysis of edges using the continuous shearlet transform. SIAM. J. Imaging Sci. 2, 959-986 (2009)

15. Guo, K., Labate, D.: Characterization of piecewise-smooth surfaces using the $3 \mathrm{D}$ continuous shearlet transform. J. Fourier Anal. Appl. 18, 488-516 (2012)

16. Guo K., Labate D.: Characterization and analysis of edges in piecewise smooth functions. Appl. Comput. Harmon. Anal. 41, 139-163 (2016)

17. Guo, K., Labate, D.: Detection of singularities by discrete multiscale directional representations. J. Geom. Anal. 28, 2102-2128 (2018)

18. Jaffard, S., Meyer, Y.: Wavelet methods for pointwise regularity and local oscillations of functions. Mem. Am. Math. Soc.,123 (1996)

19. Koh, Y.W., Lee, S.L., Tan, H.H.: Periodic orthogonal splines and wavelets. Appl. Comput. Harmon. Anal. 2, 201-218 (1995) 
20. Kutyniok, G., Labate, D.: Construction of regular and irregular shearlets. J. Wavelet Theory Appl. 1, 1-10 (2007)

21. Kutyniok, G., Labate, D.: Resolution of the wavefront set using continuous shearlets. Trans. Am. Math. Soc. 361, 2719-2754 (2009)

22. Kutyniok, G., Labate, D.: Introduction to shearlets. Appl. Comput. Harmon. Anal., Birkhäuser, pp. 1-38 (2012)

23. Kutyniok, G., Petersen, P.: Classification of edges using compactly supported shearlets. Appl. Comput. Harmon. Anal. 42, 245-293 (2017)

24. Langemann, D., Prestin, J.: Multivariate periodic wavelet analysis. Appl. Comput. Harmon. Anal. 28, 46-66 (2010)

25. Maksimenko, I.E., Skopina, M.A.: Multidimensional periodic wavelets. St. Petersbg. Math. J. 15, 165-190 (2004)

26. Mallat, S., Hwang, W.L.: Singularity detection and processing with wavelets. IEEE Trans. Inf. Theory 38, 617-643 (1992)

27. Mhaskar, H.N., Prestin, J.: On the detection of singularities of a periodic function. Adv. Comput. Math. 12, 95-131 (2000)

28. Narcowich, F.J., Ward, J.D.: Wavelets associated with periodic basis functions. Appl. Comput. Harmon. Anal. 3, 40-56 (1996)

29. Plonka, G., Tasche, M.: A Unified Approach to Periodic Wavelets. In: Chui, C.K., Montefusco, L. (eds.) Wavelets: Theory, Algorithms and Applications, Volume 5 of Wavelets Analysis and Its Applications, Academic Press, pp. 137-151 (1994)

30. Prestin, J., Selig, K.: Interpolatory and orthonormal trigonometric wavelets. In: Zeevi, J., Coifman, R. (eds.) Signal and image representation in Combined Spaces, volume 7 of Wavelets Analysis and its Applications, Academic Press, 201-255 (1998)

31. Shi, X., Zhang, H.: Determination of jumps via advanced concentration factors. Appl. Comput. Harmon. Anal. 26, 1-13 (2009)

32. Stein, E.M.: Harmonic analysis: real-variable methods, orthogonality, and oscillatory integrals. Princeton University Press (1993)

33. Stein, E.M., Weiss, G.: Introduction to Fourier analysis on Euclidean spaces. Princeton University Press (1971)

34. Tadmor, E.: Filters, mollifiers and the computation of the Gibbs phenomenon. Acta Numerica 16, 305-378 (2007)

35. Yi, S., Labate, D., Easly, G.R., Krim, H.: A shearlet approach to edge analysis and detection. IEEE Trans. Image Process. 18, 929-941 (2009)

Publisher's note Springer Nature remains neutral with regard to jurisdictional claims in published maps and institutional affiliations. 